\title{
Concerted roles of PTEN and ATM in controlling hematopoietic stem cell fitness and dormancy
}

\author{
Jerome Fortin, ' Christian Bassi,, Parameswaran Ramachandran,' Wanda Y. Li, ${ }^{1}$ Ruxiao Tian, ${ }^{1}$ Ida Zarrabi,, Graham Hill, \\ Bryan E. Snow, Jillian Haight, ${ }^{1}$ Chantal Tobin, ${ }^{1}$ Kelsey Hodgson, ${ }^{1}$ Andrew Wakeham, ${ }^{1}$ Vuk Stambolic, ${ }^{1,2}$ and Tak W. Mak \\ 1Princess Margaret Cancer Centre, University Health Network, Toronto, Ontario, Canada. ²Department of Medical Biophysics, University of Toronto, Toronto, Ontario, Canada.
}

\begin{abstract}
In order to sustain proficient life-long hematopoiesis, hematopoietic stem cells (HSCs) must possess robust mechanisms to preserve their quiescence and genome integrity. DNA-damaging stress can perturb HSC homeostasis by affecting their survival, self-renewal, and differentiation. Ablation of the kinase ataxia telangiectasia mutated (ATM), a master regulator of the DNA damage response, impairs HSC fitness. Paradoxically, we show here that loss of a single allele of Atm enhances HSC functionality in mice. To explain this observation, we explored a possible link between ATM and the tumor suppressor phosphatase and tensin homolog (PTEN), which also regulates HSC function. We generated and analyzed a knockin mouse line $\left(\right.$ Pten $\left.^{5398 A / 5398 A}\right)$, in which PTEN cannot be phosphorylated by ATM. Similar to Atm ${ }^{+/-}$, Pten $^{5398 A / 5398 A}$ HSCs have enhanced hematopoietic reconstitution ability, accompanied by resistance to apoptosis induced by genotoxic stress. Single-cell transcriptomic analyses and functional assays revealed that dormant Pten $^{5398 A / 5398 A} \mathrm{HSCs}$ aberrantly tolerate elevated mitochondrial activity and the accumulation of reactive oxygen species, which are normally associated with HSC priming for self-renewal or differentiation. Our results unveil a molecular connection between ATM and PTEN, which couples the response to genotoxic stress and dormancy in HSCs.
\end{abstract}

\section{Introduction}

Life-long hematopoiesis is required to produce all the blood cell types that maintain vital functions such as immunity, tissue repair, and oxygen transport. Hematopoietic dysregulation can have severe consequences for the organism, including impaired immune response, anemia, myelodysplastic syndromes, and leukemias (1-6). Hematopoietic stem cells (HSCs) sit at the apex of a cellular self-renewal and differentiation hierarchy that maintains hematopoiesis throughout life (7). Robust mechanisms must exist to ensure HSC fitness and integrity, thus preserving their unique capacity to self-renew and give rise to differentiating progenies (7). Accordingly, the most primitive HSCs remain largely dormant, dividing infrequently (8). Despite decades of intensive studies since their initial functional identification $(9$, 10), the mechanisms controlling whether HSCs remain dormant, self-renew, differentiate, or become senescent remain incompletely understood (7). Upon exit from dormancy, HSCs undergo profound transcriptional changes to reach an active state, primed to enter the cell cycle or differentiate (11). HSC activation is associated with enhanced metabolic activity and the production of reactive oxygen species (ROS), which can be a source of endogenous DNA-damaging stress $(11,12)$.

A proper DNA damage response is critical for maintaining HSC function $(12,13)$. The kinase ataxia telangiectasia mutated (ATM) is a master regulator of the cellular response to DNA damage (14).

Conflict of interest: The authors have declared that no conflict of interest exists. Copyright: @ 2021, American Society for Clinical Investigation.

Submitted: July 15, 2019; Accepted: January 13, 2021; Published: March 1, 2021

Reference information: J Clin Invest. 2021;131(5):e131698.

https://doi.org/10.1172/JCl131698.
ATM exerts its functions by phosphorylating a large number of targets, which in turn control specific aspects of the DNA damage response, including DNA repair, apoptosis, and cell cycle arrest (14). Genetic deletion of Atm impairs HSC function, at least in part due to the uncontrolled accumulation of ROS (15). Inactivating various ATM targets can disrupt a subset of its functions, while preserving others, sometimes resulting in opposite phenotypes to those caused by complete Atm ablation (16-20). Phosphatase and tensin homolog (PTEN) has recently been identified as being directly regulated by ATM through phosphorylation on serine 398 (S398) (21). PTEN is a tumor suppressor that controls a plethora of cellular processes (22). While many of PTEN's functions require its well-described lipid phosphatase activity at the plasma membrane, PTEN also localizes to the nucleus, where it participates in DNA repair in a lipid phosphatase-independent manner $(21,23)$. Notably, this function of PTEN involves its export from the nucleus upon phosphorylation of S398 by ATM (21). Genetic ablation of Pten in HSCs results in their uncontrolled activation, eventual exhaustion, and the development of leukemia $(24,25)$. However, whether this reflects the loss of PTEN's lipid-phosphatase activity, nuclear function, or other, remains unknown. Here, by combining the analysis of genetically engineered mouse models, single-cell transcriptomic studies, and in vivo and in vitro functional assays, we show that phosphorylation of PTEN by ATM controls HSC dormancy, response to DNA damage, and fitness.

\section{Results}

Reducing Atm genetic dosage or ablating ATM-dependent PTEN phosphorylation enhances long-term hematopoietic reconstitution potential. While investigating mechanisms linking the control of genomic stability and HSC fitness, we explored whether HSC 
A

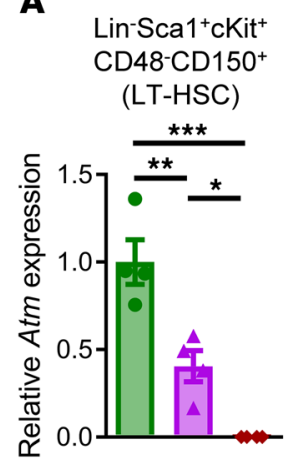

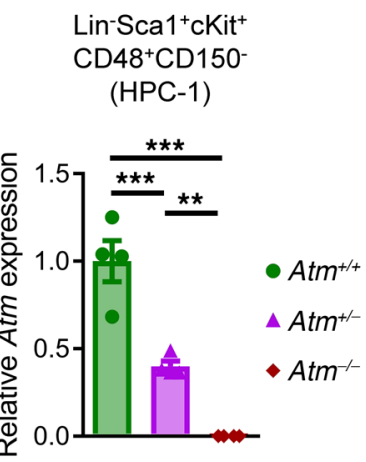

B

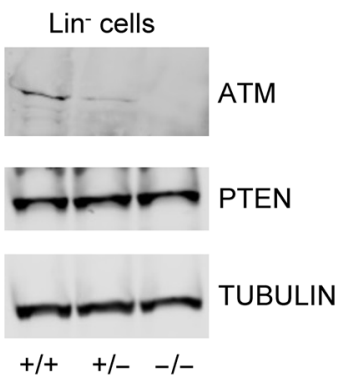

C

$\mathrm{CD} 45.2^{+} \mathrm{Atm}^{+/+}, \mathrm{Atm}^{+/-}$, or $\mathrm{Atm}^{-/-}$

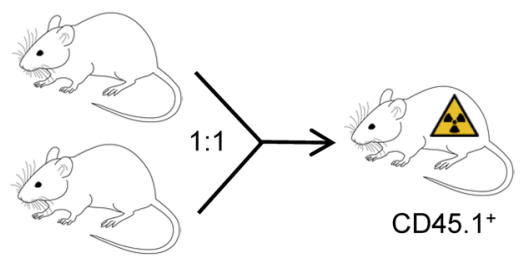

CD45.1+ (wild-type)

D

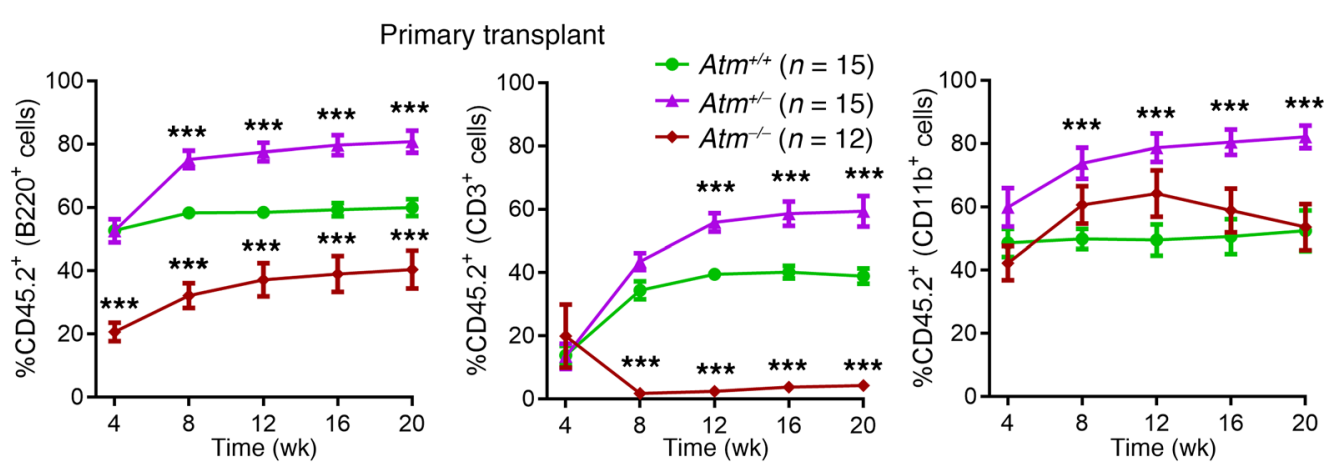

E
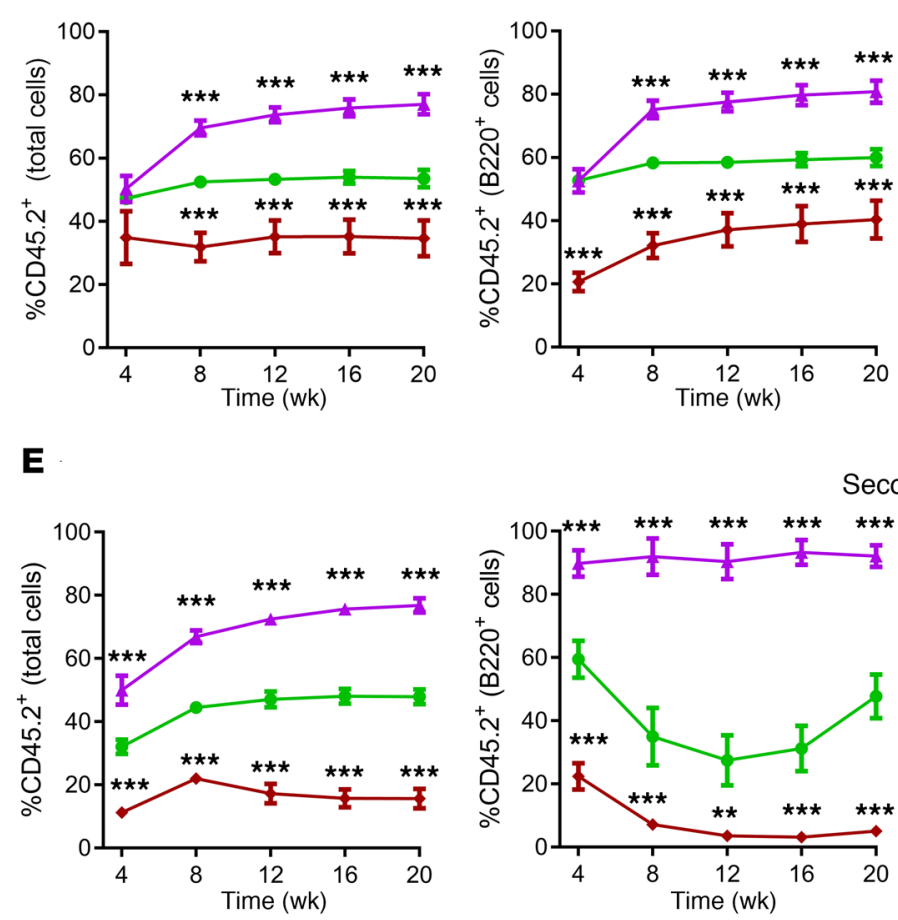

Secondary transplant
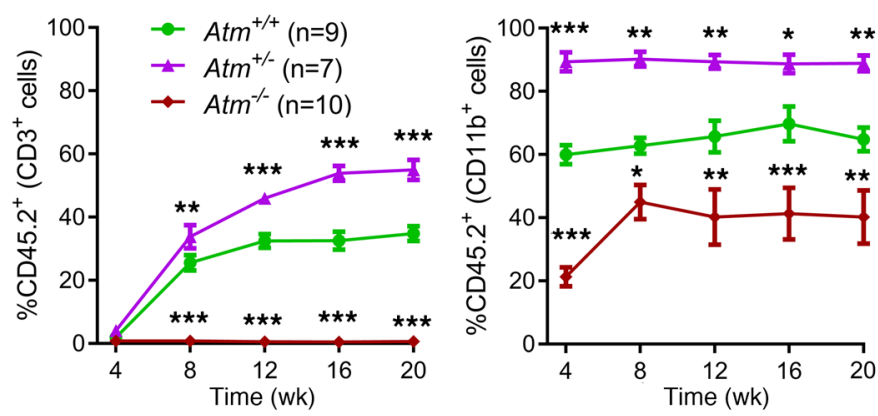

Figure 1. Enhanced competitive fitness of $\mathbf{A t m}^{+/-}$hematopoietic cells. (A) Expression of Atm, normalized to the housekeeping gene Rp/19, measured by qPCR in the indicated cell subsets isolated from $\mathrm{Atm}^{+/+}, \mathrm{Atm}^{+/-}$, and $\mathrm{Atm}^{-/-}$mice. Each symbol represents data from an individual mouse. (B) Western blot of lysates from Lin- bone marrow cells isolated from Atm ${ }^{+/+}, \mathrm{Atm}^{+/-}$, and Atm ${ }^{-/-}$mice, probed with the indicated antibodies. The same membrane was simultaneously probed with all 3 antibodies using 2 LI-COR fluorescence channels (channel 1: PTEN; channel 2: ATM, TUBULIN). See complete unedited blots in the supplemental material. (C) Diagram representing the experimental setup for competitive bone marrow transplantation experiments. (D) Proportion of total blood cells, B220+ cells, CD3+ cells, and CD11b+ cells expressing the CD45.2 marker in the peripheral blood of lethally irradiated mice transplanted with $\mathrm{Atm}^{+/+}$, Atm ${ }^{+/-}$, or $\mathrm{Atm}^{-/-} \mathrm{CD} 45.2^{+}$bone marrow cells mixed 1:1 with wild-type CD45.1+ competitors. Data are combined from 3 independent experiments in which transplanted cells were pooled from 2-3 donor animals per genotype; " $n$ " indicates the number of transplanted mice. (E) Proportion of total blood cells, B220+ cells, $\mathrm{CD}^{+}$cells, and CD11b+ cells expressing the CD45.2 marker in the peripheral blood of lethally irradiated mice secondarily transplanted with bone marrow cells isolated from the primary transplanted mice describe in panel D. Data are from 2 independent experiments; " $n$ " indicates the number of recipient mice. The gating strategy for these experiments is shown in Supplemental Figure 1, A and B. In all panels, mean and SEM are shown. ${ }^{*} P<0.05$; ${ }^{* *} P<0.01$; ${ }^{* *} P<0.001$; assessed by ANOVA with Tukey's (A) or Dunnett's (D and E; Atm ${ }^{+-}$and Atm ${ }^{-/-}$compared with Atm ${ }^{+/+}$mice) multiple-comparison test.

functionality may be sensitive to the genetic dosage of ATM, given its role in orchestrating multiple aspects of the DNA damage response. As expected, Atm expression was lower in $\mathrm{Atm}^{+/-}$hematopoietic stem and progenitor cells compared with their $\mathrm{Atm}^{+/+}$ counterparts, and was undetectable in $\mathrm{Atm}^{-/-}$cells (Figure 1A). Similar gene dosage-dependent differences in ATM protein levels were observed in lineage-negative ( (in $^{-}$) bone marrow cells (Figure 1B). To functionally evaluate their hematopoietic reconstitution ability, we competitively transplanted equal numbers of wild-type $\mathrm{CD} 45.1^{+}$and $\mathrm{Atm}^{+/+}, \mathrm{Atm}^{+/}$, or $\mathrm{Atm}^{-/-} \mathrm{CD} 45.2^{+}$bone marrow cells into lethally irradiated CD $45.1^{+}$mice (Figure 1C). Multilineage reconstitution potential was measured over 20 weeks after transplant in primary and in secondary recipients, a well-established assay to measure HSC fitness $(26,27)$. Consistent with previous results (15), $\mathrm{Atm}^{-/}$bone marrow cells were impaired in their relative ability to reconstitute long-term hematopoiesis, indicated by 
A
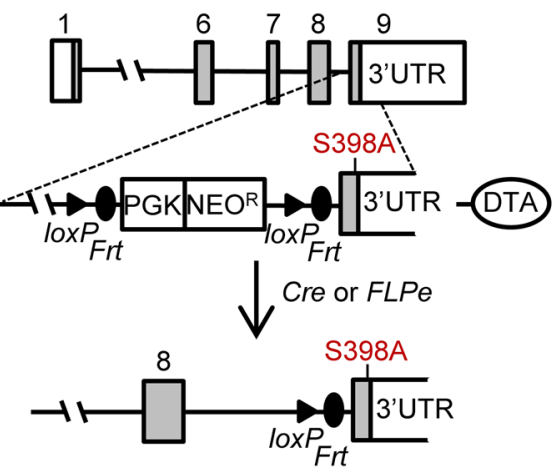

B

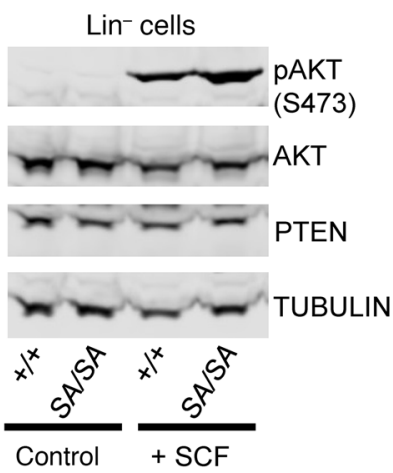

C

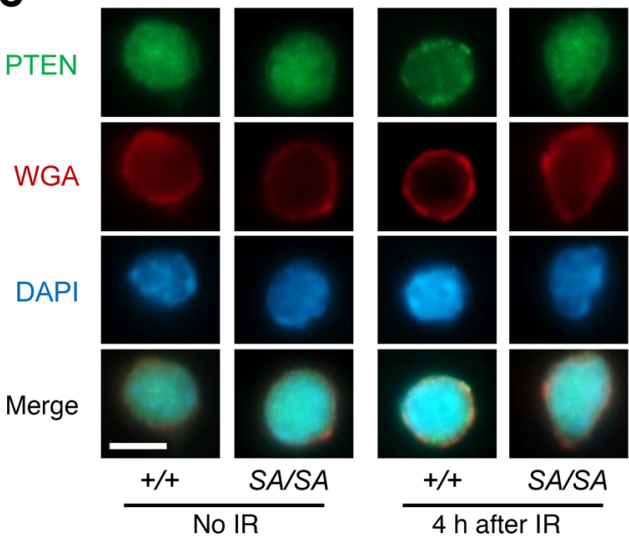

D
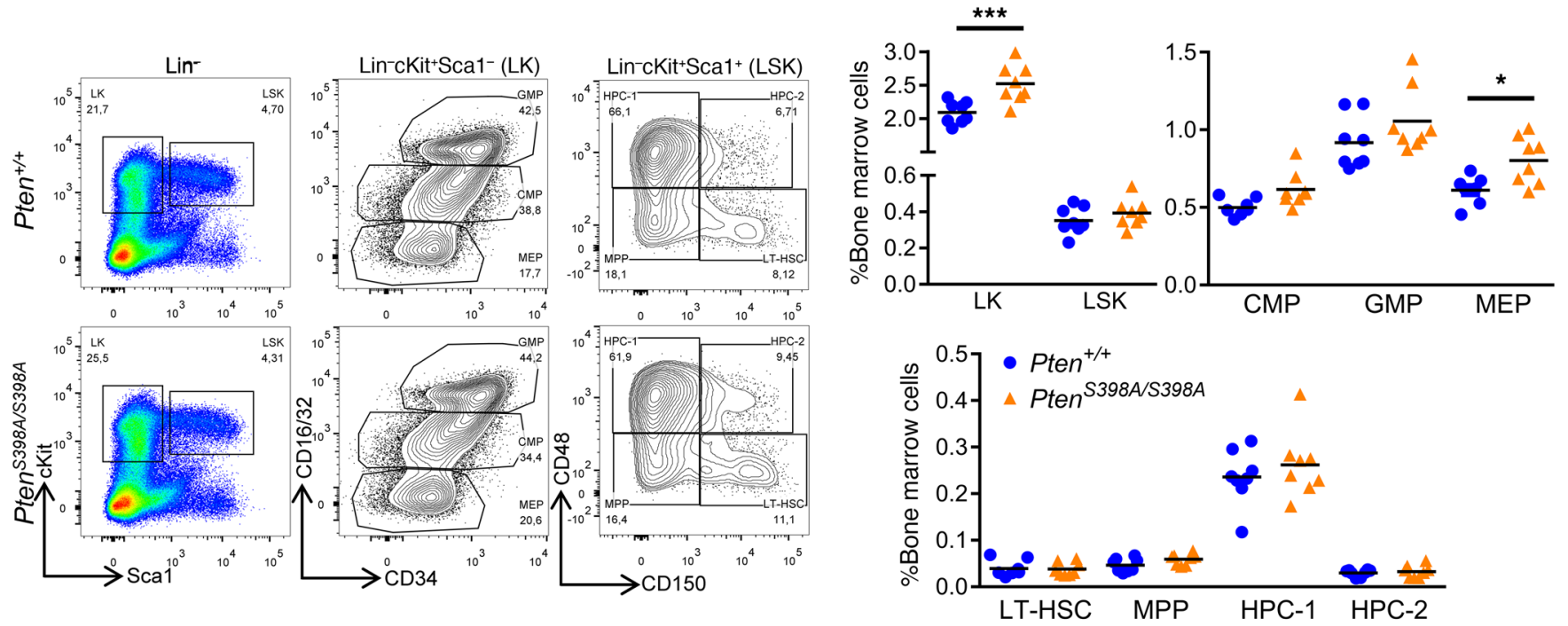

$\mathbf{E}$

CD45.2 $2^{+}$Pten $^{+/+}$or Pten ${ }^{\text {S398A/S398A }}$
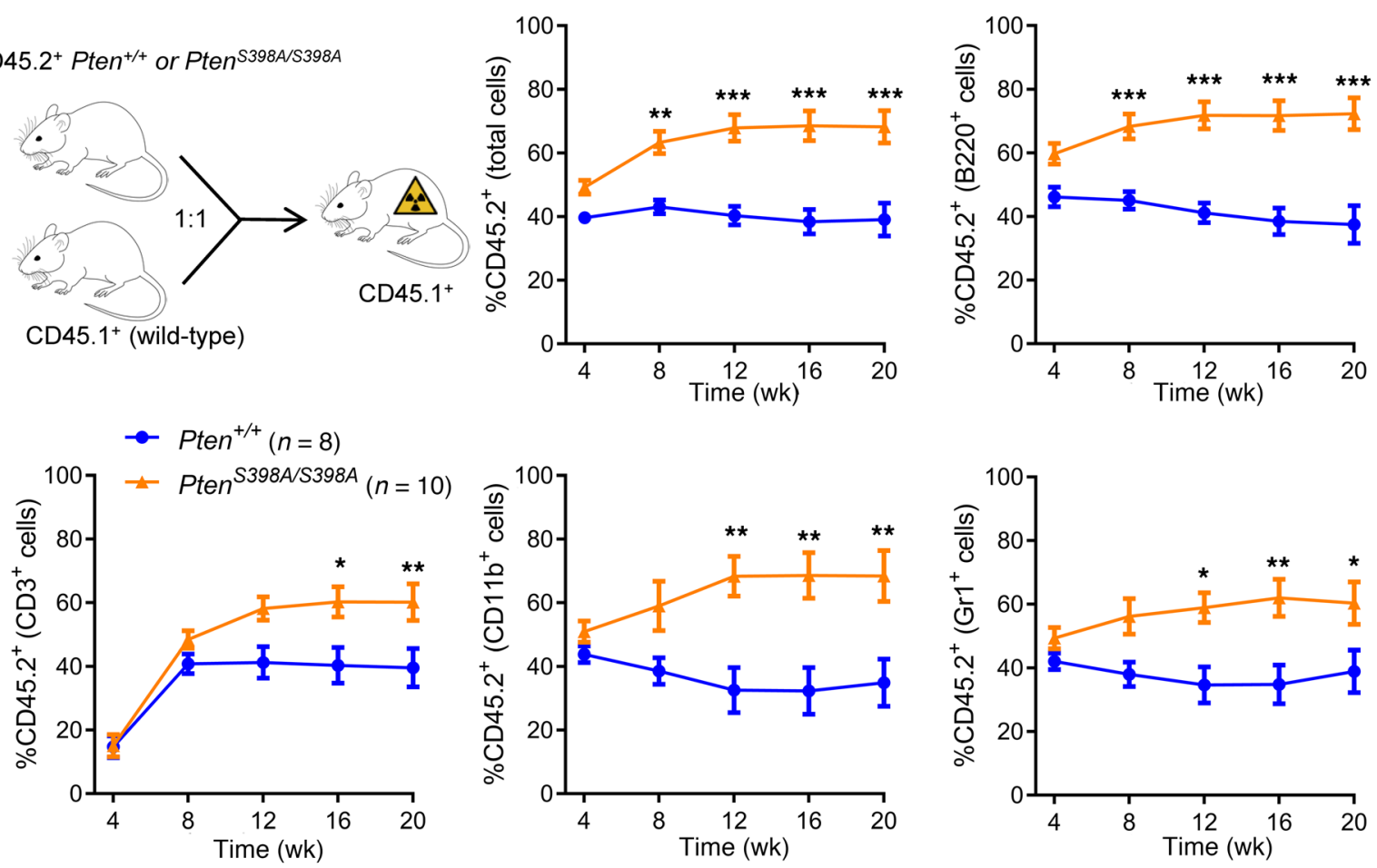
Figure 2. Enhanced competitive fitness of Pten ${ }^{5398 A / 5398 A}$ hematopoietic cells. (A) Schematic of the strategy for generating the $\operatorname{Pten}^{5398 A}$ allele, showing the Pten gene with numbered exons (top), the targeting vector (middle), and the recombined locus after excision of the selection cassette (bottom). (B) Western blot, probed with the indicated antibodies, of

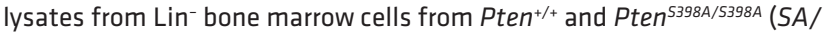
$S A$ ) mice, stimulated or not for 15 minutes with $50 \mathrm{ng} / \mathrm{mL}$ SCF. The same membrane was simultaneously probed with 4 antibodies using 2 LI-COR fluorescence channels (channel 1: AKT, PTEN; channel 2: pAKT [S473], TUBULIN). See complete unedited blots in the supplemental material. (C) Immunofluorescence microscopy images of cultured HSCs sorted from $\mathrm{Pten}^{+/+}$and Pten ${ }^{5398 A / 5398 A}$ mice, exposed or not to $1 \mathrm{~Gy}$ irradiation 4 hours prior to cell fixation. Scale bar: $5 \mu \mathrm{m}$. WCA, wheat germ agglutinin. (D) Flow cytometry plots depicting the gating strategy to identify the indicated cell populations in $\operatorname{Pten}^{+/+}$(top) and Pten ${ }^{\text {S398A/5398A }}$ (bottom) mice. The parent populations are indicated on top of the plots, and the antibodies used are indicated on the axes. Right: Quantification of the indicated cell populations in $\mathrm{Pten}^{+/+}$and $\mathrm{Pten}^{\text {S398A/5398A }}$ littermates. Each symbol represents an individual mouse. (E) Diagram representing the experimental setup, and plots showing the proportion of circulating total blood cells, B220+ cells, $\mathrm{CD}^{+}$cells, $\mathrm{CD} 11 \mathrm{~b}^{+}$cells, and $\mathrm{Cr}^{+}{ }^{+}$cells expressing the CD45.2 marker in the peripheral blood of lethally irradiated mice transplanted with Pten $^{+/+}$or Pten ${ }^{5398 A / 5398 A}$ CD $45.2^{+}$bone marrow cells mixed 1:1 with wild-type CD45.1 $1^{+}$competitors. Data are combined from 2 independent experiments in which transplanted cells were pooled from 3 donor animals per genotype; " $n$ " indicates the number of transplanted mice. In all panels, mean and SEM are shown. ${ }^{*} P<0.05$; ${ }^{* *} P<0.01$; ${ }^{* *} P$ $<0.001$; assessed by unpaired $t$ test (D) or 2-way ANOVA with Sidak's multiple-comparison test (E).

a decreased proportion of $\mathrm{Atm}^{-/}$-derived circulating blood cells, compared with their $\mathrm{Atm}^{+/+}$counterparts (Figure 1D and Supplemental Figure 1, A-C; supplemental material available online with this article; https://doi.org/10.1172/JCI131698DS1). Interestingly, this was largely restricted to the lymphoid lineages $\left(\mathrm{B} 22 \mathrm{O}^{+} \mathrm{B}\right.$ cells and $\mathrm{CD}^{+} \mathrm{T}$ cells) in primary transplantation recipients (Figure 1D and Supplemental Figure 1, A-C). The defective reconstitution potential of $\mathrm{Atm}^{-/}$cells was accentuated in secondary recipients, where it also extended to the myeloid lineages $\left(\mathrm{CD}_{11 \mathrm{~b}^{+}}\right.$and $\mathrm{Gr} 1^{+}$; Figure 1E and Supplemental Figure 1D). In stark contrast, $\mathrm{Atm}^{+/-}$ cells displayed enhanced multilineage reconstitution potential, which was maintained over the 20-week primary posttransplant period, and extended to secondary recipients, suggesting that it reflects improved HSC functionality (Figure 1, D and E, and Supplemental Figure 1, C and D).

To explain these paradoxical results, we reasoned that, while complete Atm ablation causes the loss of all ATM-regulated processes, partial Atm deficiency may preferentially impair the function of specific ATM targets. A potential role for ATMdependent regulation of PTEN in controlling HSC fitness has not yet been examined. To selectively disrupt PTEN phosphorylation by ATM in vivo, without affecting its lipid phosphatase activity, we generated a knockin allele in mice, in which serine 398 is mutated to alanine (Pten ${ }^{\text {S398A }}$ (Figure 2A). Pten ${ }^{5398 A / 3398 A}$ mice were obtained at Mendelian ratio, were viable, and developed normally.

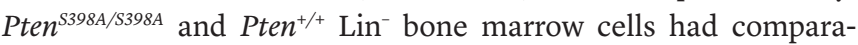
ble PTEN protein levels, as well as similar AKT phosphorylation levels with and without stem cell factor (SCF, also known as KIT ligand) stimulation (Figure 2B). Accordingly, PTEN expression was normal in $\mathrm{Atm}^{+/-}$and $\mathrm{Atm}^{-/}$hematopoietic stem and progen- itor cells (Figure 1B and Supplemental Figure 1E). Previous work has shown that in tumor cells, PTEN is excluded from the nucleus in response to irradiation (IR), through a mechanism that requires its phosphorylation by ATM (21). In cultured $\mathrm{Pten}^{+/+}$HSCs, PTEN was partially redistributed toward the plasma membrane 4 hours after 1 Gy IR, but this did not occur in Pten ${ }^{5398 A / 3398 A}, \mathrm{Atm}^{+/}$, and $\mathrm{Atm}^{-1-} \mathrm{HSCs}$ (Figure 2C and Supplemental Figure 1F).

We next investigated steady-state hematopoiesis in Pten ${ }^{5398 A / 5398 A}$ animals. Peripheral blood analyses indicated comparable circulating numbers of lymphocytes, monocytes, and red blood cells in Pten ${ }^{5398 A / 3398 A}$ and $\mathrm{Pten}^{+/+}$littermates (Supplemental Figure 1G). Pten ${ }^{S 398 A / 3398 A}$ mice had normal overall numbers of stem and progenitor cells in the bone marrow, defined as Lin$\mathrm{cKit}^{+} \mathrm{Sca} 1^{+}$(LSK) cells, but a slight expansion of $\mathrm{Lin}^{-} \mathrm{cKit}^{+} \mathrm{Sca} 1^{-}$ (LK) myeloid progenitor cells (Figure 2D). This increase appeared to be distributed between common myeloid progenitors (CMPs; $\mathrm{Lin}^{-} \mathrm{CKit}^{+} \mathrm{Sca}{ }^{-} \mathrm{CD} 34^{\mathrm{mid}} \mathrm{CD} 16 / 32^{\text {mid }}$ ), granulocyte/monocyte progenitors (GMPs; Lin $^{-} \mathrm{CKit}^{+} \mathrm{Sca}{ }^{-} \mathrm{CD} 34^{+} \mathrm{CD} 16 / 32^{+}$), and megakaryocytes/erythroid progenitors (MEPs; $\mathrm{Lin}^{-} \mathrm{CKit}^{+} \mathrm{Sca}{ }^{-} \mathrm{CD} 34^{-}$ CD16/32-) (Figure 2D). Within LSK cells, Pten ${ }^{S 398 A / 3398 A}$ mice had normal numbers of long-term HSCs (LT-HSCs; Lin ${ }^{-} \mathrm{CKit}^{+}$ $\mathrm{Sca}^{+} \mathrm{CD} 48^{-} \mathrm{CD} 150^{+}$), multipotent progenitors (MPPs; $\mathrm{Lin}^{-} \mathrm{cKit}^{+}$ $\mathrm{Sca}^{+} \mathrm{CD} 48^{-} \mathrm{CD} 150^{-}$), and hematopoietic progenitor cells (HPC-1 and HPC-2; Lin $^{-} \mathrm{CKit}^{+} \mathrm{Sca} 1^{+} \mathrm{CD} 48^{+} \mathrm{CD} 15 \mathrm{O}^{+/}$) (Figure 2D). Overall, these results suggest that steady-state hematopoiesis is largely normal in Pten $^{\$ 398 A / 3398 A}$ mice.

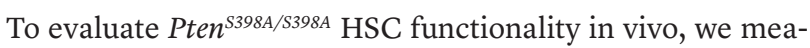
sured their relative ability to reconstitute hematopoiesis in competitive transplantation experiments. Lethally irradiated CD $45.1^{+}$ mice were transplanted with equal numbers of wild-type CD $45.1^{+}$ and Pten $^{+/+}$or Pten ${ }^{\text {S398A/3398A }}$ CD $45.2^{+}$bone marrow cells. Compared with $\mathrm{Pten}^{+/+}$, Pten ${ }^{5398 A / 3398 A}$ cells displayed enhanced multilineage reconstitution potential, as indicated by an increased proportion of Pten $^{3398 A / 3398 A}$ CD $45.2^{+}$-derived circulating blood cells (Figure 2E). This difference was maintained over the 20-week posttransplant period (Figure 2E), and further enhanced upon serial transplantation into secondary recipients (Supplemental Figure 2A). Similar to $\mathrm{Atm}^{+/}$, the enhanced reconstitution potential of Pten ${ }^{5398 A / 3398 A}$ cells was seen across $\mathrm{B}^{2} 2 \mathrm{O}^{+} \mathrm{B}$ cells, $\mathrm{CD}^{+} \mathrm{T}$ cells, and $\mathrm{CD}_{11 \mathrm{~b}^{+}}$and $\mathrm{Gr}^{+}$myeloid cells (Figure 2E and Supplemental Figure 2A), and was not associated with lineage skewing (Supplemental Figure 2B). Together, these results suggest that Pten ${ }^{5398 A / 3398 A}$ HSCs have enhanced fitness.

Pten $^{\mathrm{S398A} / 3398 A}$ HSCs are resistant to genotoxic stress. We next investigated whether the increased in vivo functionality of Pten $^{\text {S398A } 3398 A}$ HSCs might be associated with an abnormal response to genotoxic stress. We first evaluated the response of $\mathrm{Pten}^{5398 \mathrm{~A} / 3398 \mathrm{~A}}$ mice to a 10 Gy IR challenge, which normally causes lethality as a result of hematopoietic failure (10). Whereas all of the $\mathrm{Pten}^{+/+}$ mice succumbed following IR with a median survival of 13.5 days, lethality was delayed in their Pten ${ }^{5398 A / 3398 A}$ littermates, with half of the animals surviving until at least 30 days after IR (Figure 3A). This was reminiscent of the phenotype observed in mice lacking the ATM target CHK2, which regulates p53-dependent cell cycle arrest and apoptosis in response to DNA damage $(20,28)$. Therefore, we sorted LT-HSCs and progenitors from $\mathrm{Pten}^{+/+}$and Pten $^{\text {S398A/3398A }}$ littermates, and measured their propensity to under- 
A

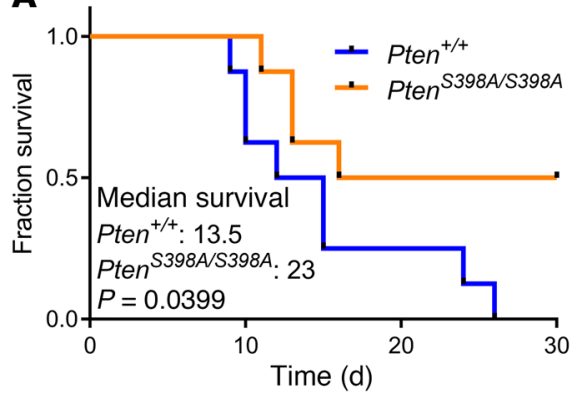

C
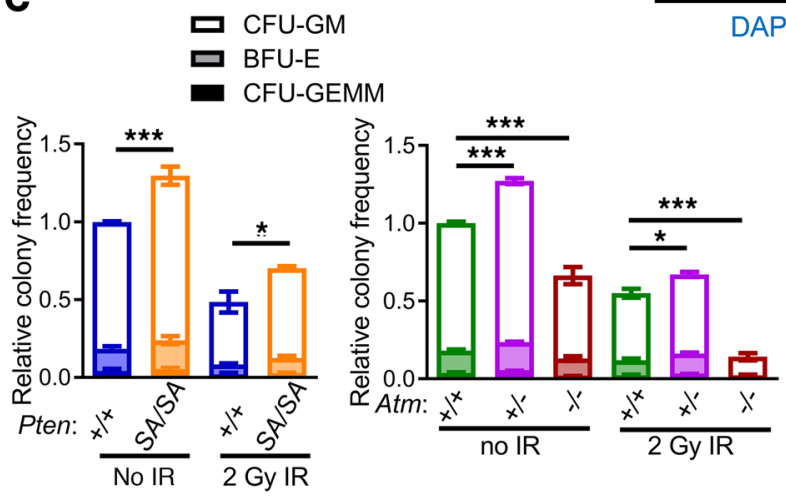

B

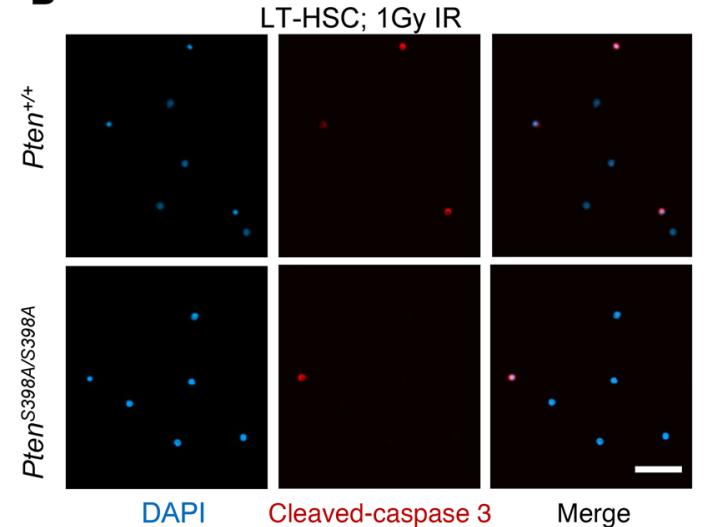

D

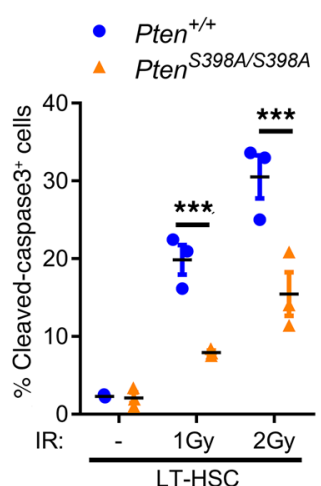

E

Foci:
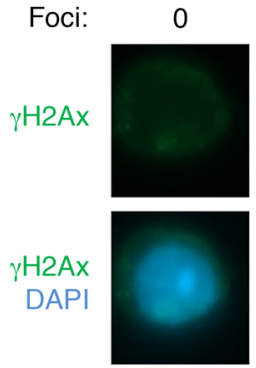

Foci:

0
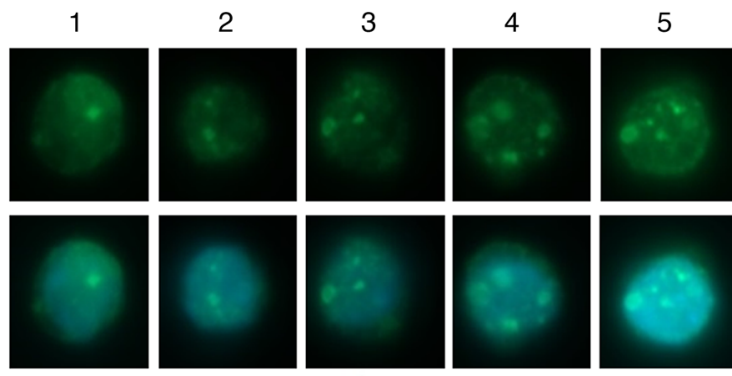

$\mathbf{F}$
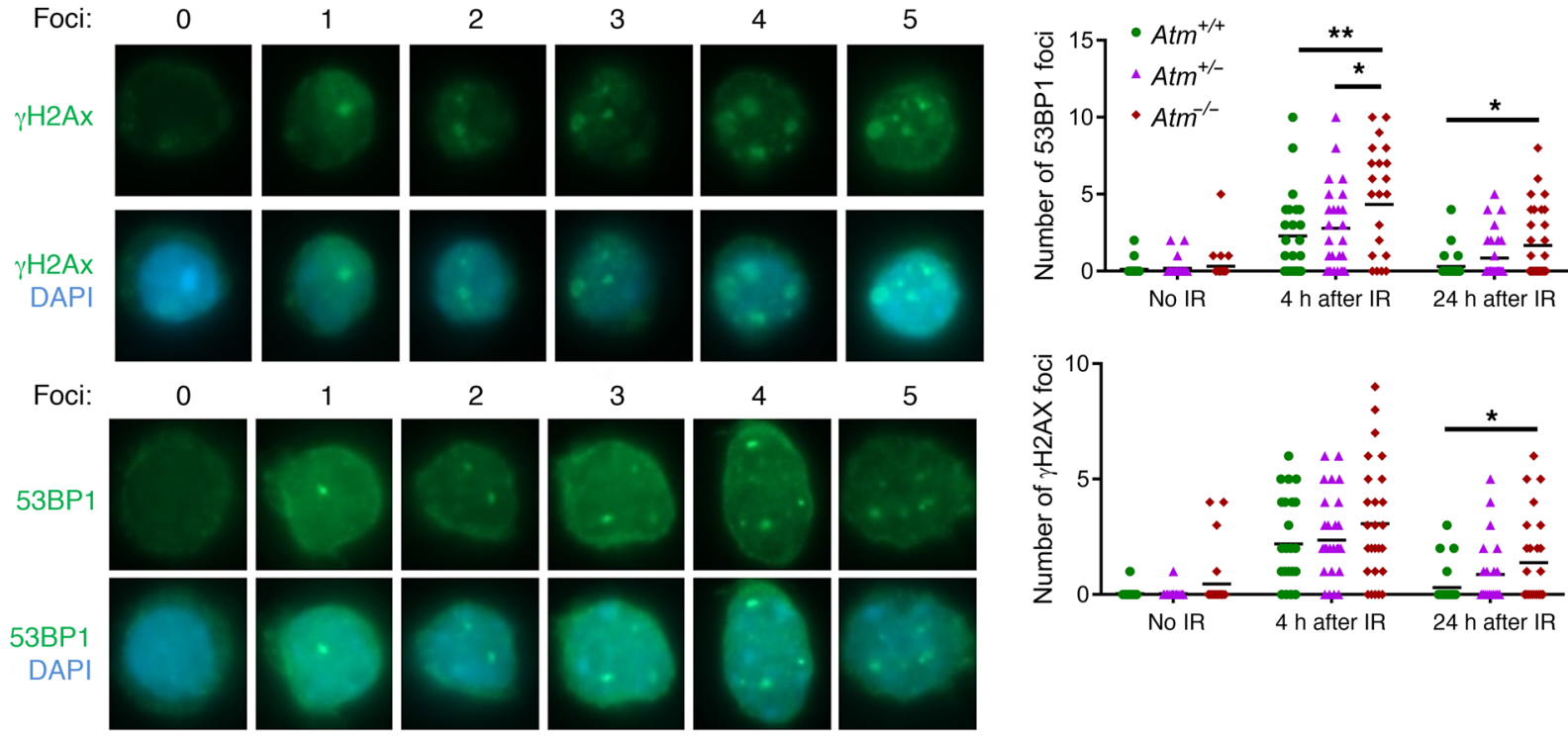

4

5
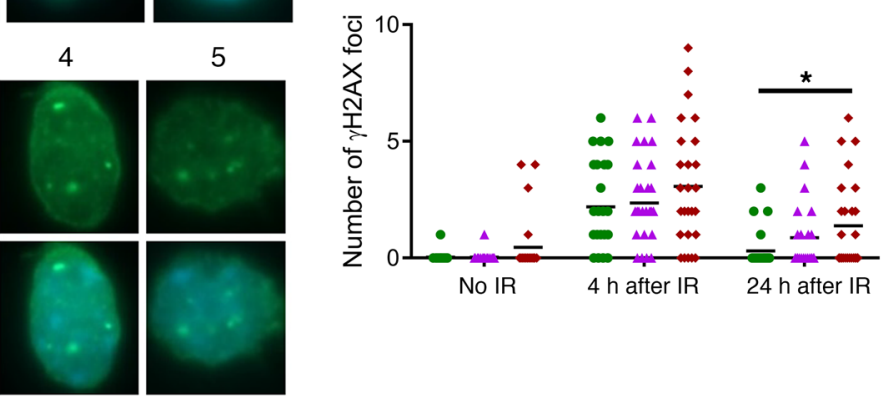

G
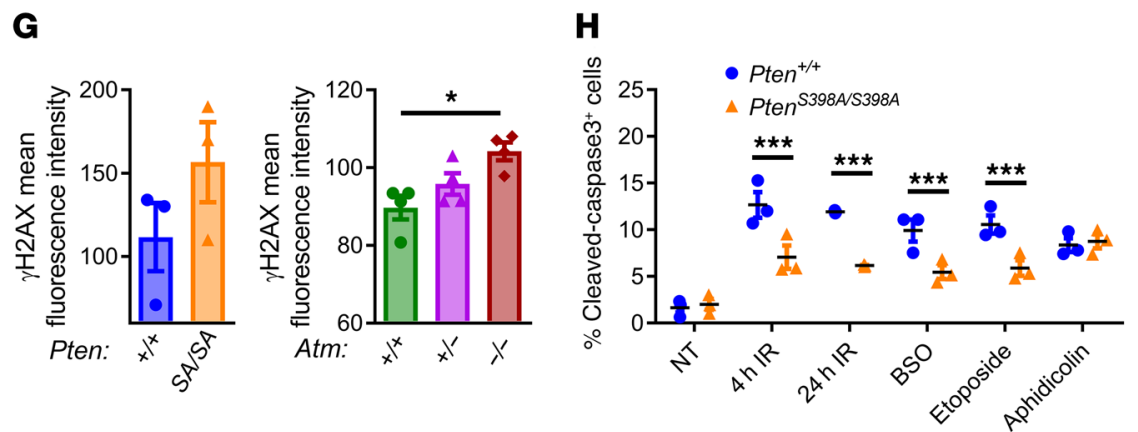

I
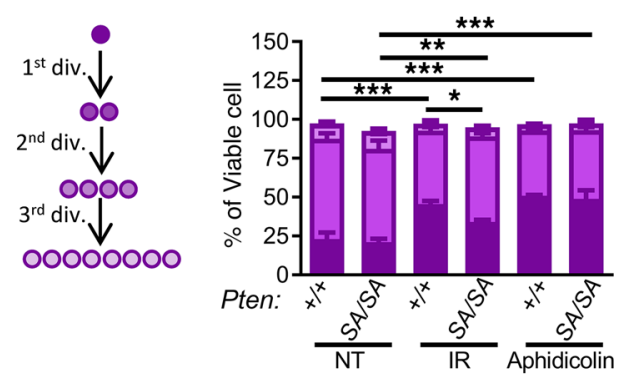
Figure 3. Pten ${ }^{5398 A / 5398 A}$ hematopoietic stem cells are resistant to genotoxic stress. (A) Kaplan-Meier curve depicting the survival of $\operatorname{Pten}^{+/+}$and Pten ${ }^{5398 A / 5398 A}$ mice in response to $10 \mathrm{~Gy}$ full-body irradiation. (B) Immunofluorescence images and quantification of cleaved caspase 3-positive LT-HSCs from Pten ${ }^{+/+}$and Pten ${ }^{5398 A / 5398 A}$ mice, 4 hours after 1 or 2 Gy irradiation. $n=3$ experiments. Scale bar: $50 \mu \mathrm{m}$. (C) Relative frequencies of hematopoietic colonies formed by bone marrow cells with the indicated genotypes in M3434 methylcellulose medium, with or without 2 Gy irradiation. $n=3$ experiments. Significance symbols indicate differences in overal colony numbers. CFU-GM, granulocyte/macrophage colony-forming unit; BFU-E, burst-forming unit, erythroid; CFU-GEMM, granulocyte/erythrocyte/monocyte/megakaryocyte colony-forming unit. (D) Number of 53BP1 and $\gamma \mathrm{H} 2 \mathrm{AX}$ foci in $\mathrm{Pten}^{+/+}$and $\mathrm{Pten}^{5398 A / 5398 A}$ LT-HSCs, subjected or not to irradiation (IR). Each dot represents an individual cell. (E) Immunofluorescence images of LT-HSCs with the indicated numbers of 53BP1 or $\gamma \mathrm{H} 2 \mathrm{AX}$ foci. (F) Number of 53BP1 and $\gamma \mathrm{H} 2 \mathrm{AX}$ foci in $\mathrm{Atm}^{+/+}, \mathrm{Atm}^{+/}$, and $\mathrm{Atm}^{-/}$ LT-HSCs, subjected or not to irradiation (IR). Each symbol represents an individual cell. (G) $\gamma \mathrm{H} 2 \mathrm{AX}$ immunoreactivity, measured by flow cytometry, in LT-HSCs isolated from mice with the indicated genotypes. Each symbol represents an individual mouse. (H) Proportion of cleaved caspase 3-positive cells, assessed by flow cytometry, in cultured Pten $^{+/+}$and $P$ ten $^{5398 A / 5398 A}$ LT-HSCs treated as indicated: not treated (NT), 1 Gy irradiation (IR), $100 \mu \mathrm{M}$ buthionine sulfoximine (BSO), $0.25 \mu \mathrm{M}$ etoposide, or $50 \mathrm{ng} / \mathrm{mL}$ aphidicolin. $n=3$ experiments. (I) Number of cell divisions (div.) undergone by Pten $^{+/+}$ and Pten ${ }^{5398 A / 5398 A}$ LT-HSCs after 72 hours of culture without treatment (NT), following 2 Gy irradiation (IR), or with $50 \mathrm{ng} / \mathrm{mL}$ aphidicolin. $n=3$ experiments. In all panels, mean and SEM are shown. ${ }^{*} P<0.05 ;{ }^{* *} P<$ 0.01 ; ${ }^{* *} P<0.001$; assessed by log-rank (Mantel-Cox) test (A) or 2-way ANOVA with Sidak's (B and $\mathbf{H})$, Dunnett's (C, with Pten $^{+/+}$and Atm $^{+/+}$being the control groups), or Tukey's (F, G, and I) multiple-comparison test.

go apoptosis upon an IR challenge in vitro, as judged by the accumulation of cleaved caspase 3. Four hours after $1 \mathrm{~Gy}$ or $2 \mathrm{~Gy} \mathrm{IR,}$ Pten ${ }^{5398 A / 3398 A}$ LT-HSCs and progenitors had a lower proportion of cells expressing cleaved caspase 3 compared with $\mathrm{Pten}^{+/+}$cells, suggesting a defective apoptotic response to DNA damage (Figure 3B and Supplemental Figure 3A). Accordingly, the colony-forming ability of Pten ${ }^{3398 A / 3398 A}$ bone marrow cells in methylcellulose was enhanced, both under baseline conditions and in response to $2 \mathrm{~Gy}$ IR (Figure 3C). This was also observed with $\mathrm{Atm}^{+/}$cells, whereas $\mathrm{Atm}^{-1-}$ cells were profoundly impaired in their ability to generate colonies, particularly after IR (Figure 3C).

To assess whether the apparent resistance of Pten ${ }^{3398 A / 3398 A}$ cells to IR was associated with an abnormal response to DNA damage or its repair, we measured the emergence and resolution of $\gamma \mathrm{H} 2 \mathrm{AX}$ and 53BP1 foci following ex vivo IR (1 Gy) in sorted LT-HSCs. $\mathrm{Pten}^{5398 \mathrm{~A} / 398 \mathrm{AA}}$ and $\mathrm{Pten}^{+/+}$cells accumulated similar numbers of foci 4 hours after IR, and these were largely resolved by 24 hours (Figure 3, D and E). In contrast, $\mathrm{Atm}^{-/}$cells showed more $\gamma \mathrm{H} 2 \mathrm{AX}$ and 53BP1 foci at 4 hours, and were impaired in damage resolution at 24 hours after IR (Figure 3F). Pten ${ }^{3398 A / 3398 A}$ and $\mathrm{Atm}^{+/-}$cells tended to retain more foci at the 24 -hour time point, such that $\mathrm{Atm}^{+/}$cells were not significantly different than either the $\mathrm{Atm}^{+/+}$ or $\mathrm{Atm}^{-1-}$ LT-HSCs (Figure 3F). Similarly, assessment of $\gamma \mathrm{H} 2 \mathrm{AX}$ immunoreactivity in freshly isolated bone marrow cells pointed to an accumulation of endogenous DNA damage by $\mathrm{Atm}^{-1}$ LT-HSCs in vivo, whereas Pten ${ }^{5398 A / S 398 A}$ and $\mathrm{Atm}^{+/}$cells showed nonsignificant trends compared with their respective wild-type controls (Figure 3G). To assess whether Pten ${ }^{5398 A / 3398 A}$ LT-HSCs had similar responses to different types of DNA damage, we measured $\gamma \mathrm{H} 2 \mathrm{AX}$ and 53BP1 foci, as well as cleaved caspase 3 immunoreactivity, 4 hours and 24 hours following IR, or upon topoisomerase II poisoning (etoposide), oxidative stress (buthionine sulfoximine; BSO), or replication stress (aphidicolin). Interestingly, Pten ${ }^{S 398 A / 3398 A}$ cells were resistant to IR-, etoposide-, and BSO-induced apoptosis, but had normal sensitivity to aphidicolin (Figure $3 \mathrm{H}$ and Supplemental Figure 3B). Induction of $\gamma \mathrm{H} 2 \mathrm{AX}$ and 53BP1 foci in response to all treatments was similar in Pten ${ }^{5398 A / / 3398 A}$ and Pten $^{+/+}$LT-HSCs (Supplemental Figure 3C). Overall, these results suggest that Pten ${ }^{5398 A / 3398 A}$ cells have a higher tolerance to the toxic effects of certain types of DNA damage.

Genotoxic stress can induce cell cycle arrest, a process that is regulated by ATM (14). We therefore assessed whether LT-HSCs, which are predominantly quiescent, had an altered cell cycle distribution in Pten $^{\text {S398A/3398A }}$ mice. The proportion of freshly isolated LT-HSCs in G phase was slightly lower in Pten ${ }^{5398 A / / 3988 A}$ mice compared with their $\mathrm{Pten}^{+/+}$littermates, with a concomitant increase in the proportion of S phase-primed $\mathrm{G}_{1}$ cells (Supplemental Figure 3, D and E). The cell cycle distribution in more differentiated progenitor subsets was similar between genotypes (Supplemental Figure 3E). Interestingly, this mild loss of quiescence of Pten ${ }^{3398 A / 3398 A}$ LT-HSCs under homeostatic conditions was also observed in $\mathrm{Atm}^{+/}$, but not in $\mathrm{Atm}^{-/}$animals (Supplemental Figure 3F). To evaluate whether Pten ${ }^{3398 A / 3398 A}$ LT-HSCs are deficient in DNA damage-induced cell cycle arrest, we sorted LT-HSCs from $\mathrm{Pten}^{5398 \mathrm{~A} / 3398 \mathrm{~A}}$ and $\mathrm{Pten}^{+/+}$littermates, pulsed them with a fluorescent cell division tracer ex vivo, exposed them to IR or aphidicolin, and subjected them to short-term culture to allow 1 or 2 cell divisions (Figure 3I and Supplemental Figure 3G). The proportion of cells having divided was decreased by either IR or aphidicolin (Figure 3I). Compared with Pten $^{+/+}$, Pten ${ }^{3398 A / / 3989 A}$ LT-HSCs were less sensitive to the effect of IR (Figure 3I). In contrast, cells with either genotype responded similarly to aphidicolin (Figure 3I).

To evaluate the functional consequences of the enhanced tolerance of Pten $n^{5398 A / 3398 A}$ HSCs to DNA damage, we performed competitive bone marrow transplantation following an ex vivo IR challenge (29). Pten $^{+/+}$or Pten ${ }^{\text {S39AA/3398A }} \mathrm{CD} 45.2^{+}$bone marrow cells were subjected to $2 \mathrm{~Gy} \mathrm{IR}$, and mixed at a 10:1 ratio with nonirradiated wild-type $\mathrm{CD} 45.1^{+}$cells prior to transplantation into lethally irradiated CD45.1 $1^{+}$recipients (Figure 4A). Compared with $\mathrm{Pten}^{+/+}$, $\mathrm{Pten}^{5398 A / 3398 \mathrm{CD}} \mathrm{CD} 4.2^{+}$cells displayed a competitive advantage that was maintained over 20 weeks following transplantation (Figure 4A and Supplemental Figure 4A), and sustained in secondary recipient mice (Supplemental Figure 4B). This enhanced reconstitution potential was seen across multiple mature cell types (Figure $4 \mathrm{~A}$ and Supplemental Figure 4A) and was not associated with preferential expansion of a particular lineage (Supplemental Figure 4C). To examine the HSC and progenitor reconstitution potential of irradiated $\mathrm{Pten}^{+/}$and $\mathrm{Pten}^{\mathrm{S398A} / 3398 A}$ cells, we analyzed the bone marrow of recipient mice 20 weeks after transplantation. Compared with recipients of $\mathrm{Pten}^{+/+}$cells, animals transplanted with $\mathrm{Pten}^{\mathrm{S398A} / / 3988 \mathrm{~A}}$ cells had a higher proportion of CD $45.2^{+}$LT-HSCs (Figure 4B and Supplemental Figure 4D). Interestingly, within the CD $45.2^{+}$LSK compartment, Pten $^{\text {S398A } / 3398 A}$ bone marrow recipients had a higher proportion of LT-HSCs, and a lower proportion of more differentiated HPC-2 cells (Figure 4C). By contrast, the proportion of these subsets within CD $45.1^{+}$LSK cells was similar between conditions, as expected given that $\mathrm{CD} 45.1^{+}$competitor cells were wild type, 
A
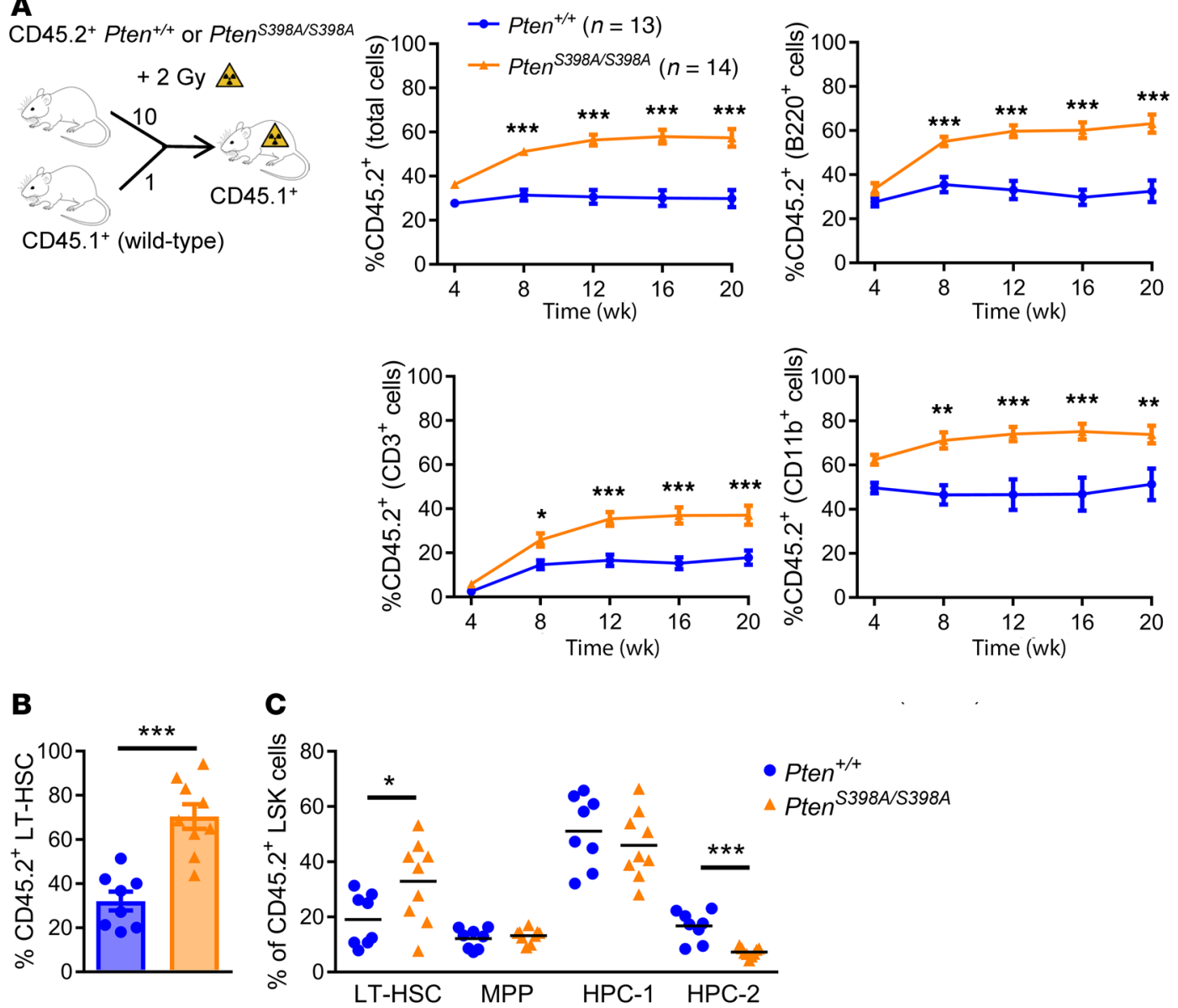

Figure 4. Improved long-term hematopoietic reconstitution ability of irradiated Pten ${ }^{5398 A / 5398 A}$ bone marrow cells in vivo. (A) Diagram representing the experimental setup, and plots showing the proportion of circulating total blood cells, B220+ ${ }^{+}$cells, CD3 ${ }^{+}$cells, and CD11 b ${ }^{+}$cells expressing the CD45.2 marker in the peripheral blood of lethally irradiated mice transplanted with Pten ${ }^{+/+}$or Pten ${ }^{5398 A / 5398 A}$ CD $45.2^{+}$bone marrow subjected to 2 Gy irradiation and mixed 10:1 with nonirradiated wild-type CD45.1+ competitors. Data are combined from 2 independent transplantation experiments. In each experiment, transplanted cells were pooled from 3 donor animals per genotype; " $n$ " indicates the number of transplanted mice. (B) Proportion of LT-HSCs expressing CD45.2 in the bone marrow from mice transplanted as depicted in panel A, 20 weeks after transplantation. Each symbol represents an individual mouse. (C) Proportion of the indicated stem and progenitor cell populations within CD45.2-expressing LSK cells in the bone marrow from mice transplanted as in panel A, analyzed 20 weeks after transplantation. Each symbol represents an individual mouse. The gating strategy is depicted in Supplemental Figure 4D. In all panels, mean and SEM are shown. ${ }^{*} P<0.05$; ${ }^{* *} P<0.01$; ${ }^{* *} P<0.001$; assessed by 2-way ANOVA with Sidak's multiple-comparison test (A) or $t$ test (B and $\mathbf{C})$.

and were derived from the same pool of donors for all the transplanted mice (Supplemental Figure 4E). Overall, these results suggest that $P t e n^{5398 A / / 3398 A}$ HSCs preserve enhanced survival and fitness in response to DNA damage in vitro and in vivo.

Transcriptomic anomalies in single quiescent Pten ${ }^{5398 A / 3398 A}$ HSCs. To begin deciphering the molecular mechanisms underlying enhanced HSC functionality in Pten ${ }^{33984 / 3398 A}$ mice, we profiled the transcriptomes of sorted LT-HSCs by single-cell RNA sequencing (RNA-seq), using the microfluidic chip-based Fluidigm C1 platform (see Supplemental Figure 5A for sorting strategy). After quality control filtering (see Supplemental Methods), we obtained transcriptomic data for $116 \mathrm{Pten}^{+/+}$and $90 \mathrm{Pten}^{\text {S398A/S398A }}$ cells, derived from a total of 6 mice from 2 independent sorting procedures for each genotype (Supplemental Figure 5B). Clustering of the single cells using the Seurat pipeline (30) and visualization by $\mathrm{t}$-distributed stochastic neighbor embedding (t-SNE) identified 3 cell clusters (Clusters 1, 2, and 3; Figure 5A). Marker gene analy- sis and machine learning-based cell-cycle phase assignment (31) indicated that Cluster 3 contained actively proliferating $\left(S, G_{2}\right.$, or $\mathrm{M}$ phase) cells, as indicated by high expression of genes such as $m$ Ki67, Top2a, Ccnb2, and Cdca3 (Supplemental Figure 5C). Clusters 1 and 2 represented quiescent HSCs, highlighted by low expression of cell-cycle regulators, and high expression of quiescent HSC markers such as Ly6a, Mllt3, Sult1a1, and Hlf (Supplemental Figure 5C and refs. 11, 32, 33). Interestingly, the $\mathrm{Pten}^{++}$and Pten ${ }^{5398 A / 3398 A}$ cells markedly differed in their distribution among the 2 quiescent cell clusters, being enriched in Cluster 2 and Cluster 1, respectively (Figure 5A and Supplemental Figure 5D). Therefore, we sought to identify transcriptional differences between Pten $^{+/+}$and Pten ${ }^{S 398 A / 3398 A}$ quiescent HSCs.

Recently, quiescent HSCs have been shown to undergo a transition from a dormant to an active state under homeostatic conditions $(8,11)$. We reclustered quiescent HSCs (Clusters 1 and 2 from the above analysis), based on a gene signature that defines 

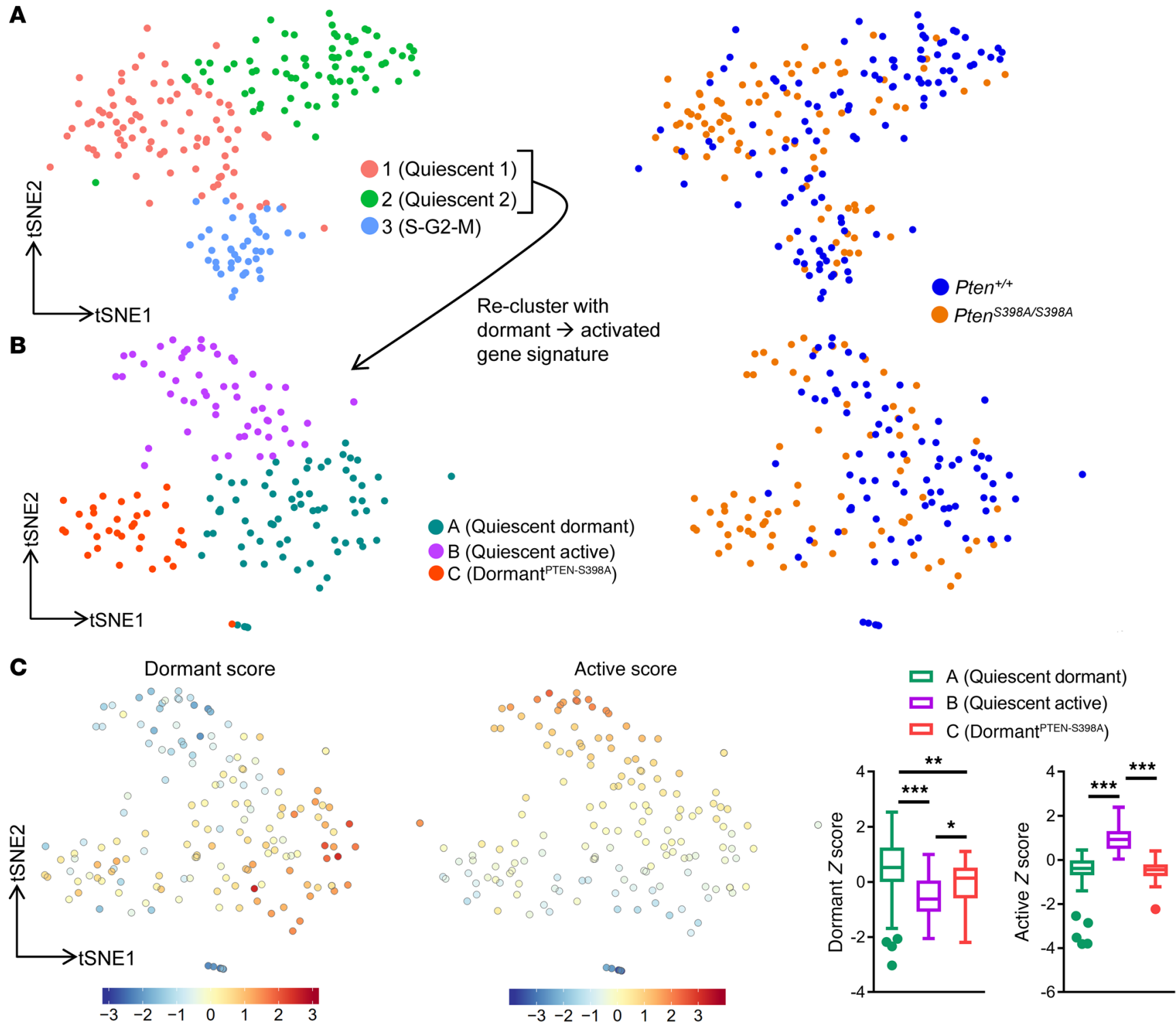

D

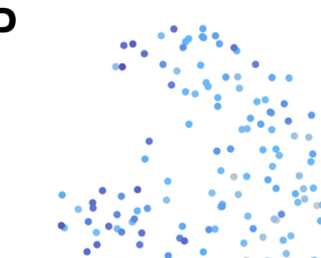

Ndufa4
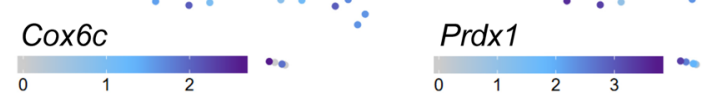

Atp5h

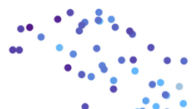

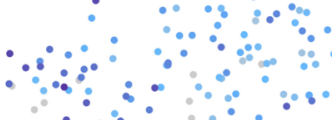

Txn1

Sesn1

$\operatorname{Trp53}$

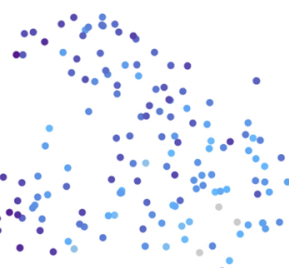

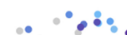
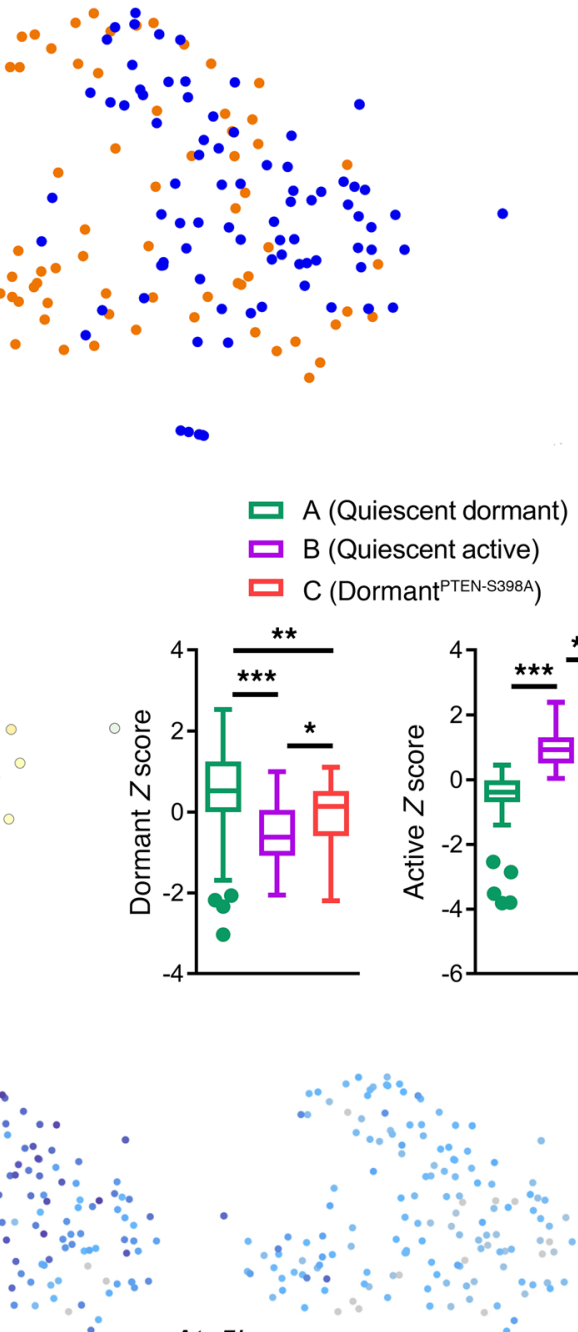

$1 \quad 2$
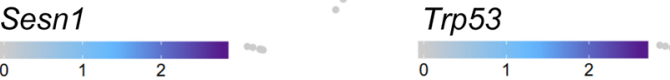

Procr 
Figure 5. Altered transcriptome of quiescent Pten ${ }^{5398 A / 5398 A}$ hematopoietic stem cells. (A) t-SNE representation of single HSCs sorted from $\mathrm{Pten}^{+/+}$ and $P \operatorname{ten}^{5398 A / 5398 A}$ mice, and analyzed by RNA sequencing. Cells are colored to represent Seurat-identified clusters (left) or genotype (right). (B) t-SNE representation of quiescent Pten $^{+/+}$and Pten ${ }^{5398 A / 5398 A}$ HSCs (cell in Clusters 1 and 2 from panel $\mathbf{A}$ ), reclustered based on a gene signature that defines the transition from the dormant to active state. Cells are colored to represent Seurat-identified clusters (left), or genotype (right). (C) Left: Same plots as in panel B, but showing the $z$-scored weighted average expression of genes defining the dormant (left) or active (right) states. Right: Quantification of the dormant and active $z$ scores in each of the Seurat-identified cell clusters represented in panel B, represented as box plots with Tukey whiskers. Data were analyzed using 1-way ANOVA with Tukey's multiple-comparison test. ${ }^{*} P<0.05 ;{ }^{* *} P<0.01$; ${ }^{* *} P<0.001$. (D) Relative expression (z-scored) of selected genes in single quiescent $P$ ten ${ }^{+/+}$ and Pten ${ }^{5398 A / 5398 A}$ HSCs, overlaid on the t-SNE plots shown in panel $\mathbf{B}$.

the transition from the dormant to the active state in single-cell RNA-seq analyses (11). This procedure allocated quiescent $\mathrm{Pten}^{+/+}$ and Pten ${ }^{5398 A / 3398 A}$ HSCs to 3 new clusters (Clusters A, B, and C; Figure 5B). Pten ${ }^{+/+}$cells were largely restricted to 2 clusters (A and B) (Figure 5B and Supplemental Figure 6A). To functionally annotate these clusters, we computed scores measuring the expression of genes that characterize dormant and active HSCs (Figure 5C). Cluster B cells could be assigned to the active state, as reflected by elevated expression of genes involved in DNA replication and nucleotide synthesis such as Pcna, Mcm4, Mcm6, and Dtymk (Supplemental Figure 6B and ref. 11). Gene set enrichment analysis (GSEA) confirmed this classification (Supplemental Figure 6C). Similar proportions of $\mathrm{Pten}^{+/+}$and Pten ${ }^{S 398 A / 3398 A}$ cells were assigned to this active cell cluster, suggesting that the ability of

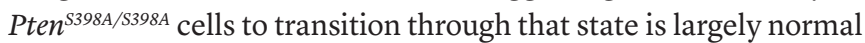
(Figure 5B and Supplemental Figure 6A). Cells in Cluster A displayed high dormant and low active scores, and therefore could readily be assigned to the dormant state (Figure 5C). These cells showed particularly high expression of quiescent HSC markers such as Ly6a, Mllt3, Sult1a1, and Hlf (Supplemental Figure 6B) (11, 32, 33). Importantly, Pten ${ }^{3398 A / 3398 A}$ cells were underrepresented in Cluster A compared with $\mathrm{Pten}^{+/+}$cells, and made up almost the entirety of Cluster C (Figure 5B and Supplemental Figure 6A). Cells in Cluster C showed a low active score similar to Cluster A, but an intermediate dormant score that fell between that of Cluster A and Cluster B cells (Figure 5C). Together with an underrepresentation of Pten ${ }^{5398 A / 3398 A}$ cells in the dormant Cluster A, these data suggest that Cluster $C$ represents an altered dormant state (hereafter, "dormant ${ }^{\mathrm{PTEN}-\mathrm{S} 398 \mathrm{~A} \text { "). }}$

Pten ${ }^{5398 A / S 398 A}$ dormant HSCs tolerate elevated levels of oxidative stress and have improved fitness. To understand how dormant ${ }^{\mathrm{PTEN}-\mathrm{S3} 38 \mathrm{~A}}$ HSCs differ from their dormant counterparts, we
A
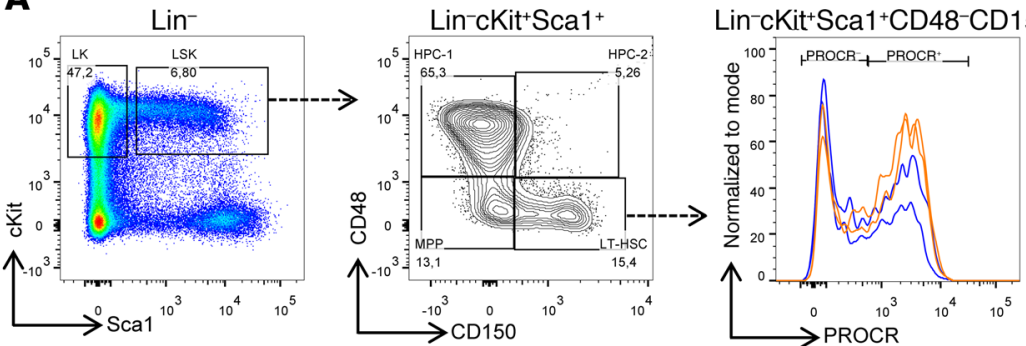

B

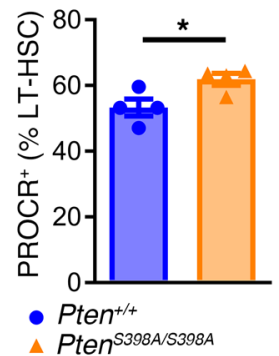

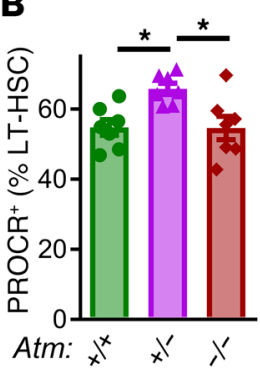

C

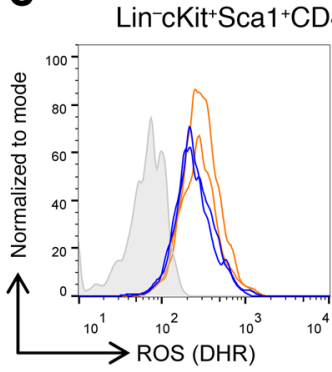

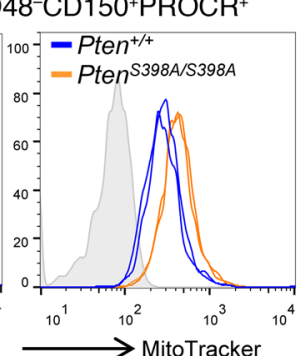

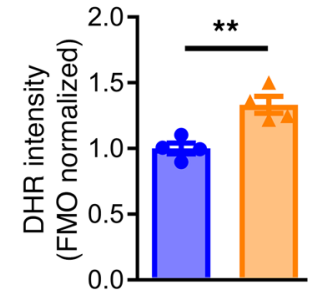

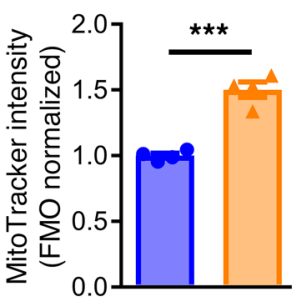

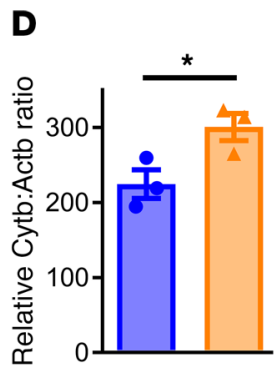

Figure 6. Pten ${ }^{5398 A / 5398 A}$ hematopoietic stem cells tolerate elevated levels of oxidative stress. (A) Flow cytometry plots depicting the gating strategy to identify Lin ${ }^{-} \mathrm{Kit}^{+}{ }^{-} \mathrm{Sca1}^{-}$cells (LK), Lin-cKit ${ }^{+}$Sca1+ cells (LSK), long-term hematopoietic stem cells (LT-HSCs), multipotent progenitors (MPPs), 2 subsets of hematopoietic progenitors (HPC-1 and HPC-2), and PROCR LT-HSCs in Pten ${ }^{+/+}$and Pten $^{5398 A / 5398 A}$ mice. The parent populations are indicated on top of the plots, and the antibodies used are indicated on the axes. In the PROCR staining histogram, blue lines indicate cells from Pten ${ }^{+/+}$mice, and orange lines indicate cells from Pten ${ }^{5398 A / 5398 A}$ littermates. Quantification of LT-HSCs expressing PROCR is shown at the right. Each symbol represents an individual animal. (B) Quantification of LT-HSCs expressing PROCR in Atm ${ }^{+/}, \mathrm{Atm}^{+/-}$, and $\mathrm{Atm}^{-/-}$littermates. Each symbol represents an individual mouse. (C) Flow cytometry plots (left) and quantification (right) of fluorescence intensity for dihydrorhodamine (DHR), an indicator of reactive oxygen species (ROS) (left) and for MitoTracker Green, an indicator of mitochondrial content (right) in PROCR ${ }^{+}$LT-HSCs in Pten ${ }^{+/+}$and Pten ${ }^{5398 A / 5398 A}$ mice. Each symbol represents an individual animal. Gray traces show the background fluorescence intensity in the absence of the DHR and MitoTracker dyes. (D) Relative levels of mitochondrial over nuclear DNA, assessed by qPCR measurements of Cytb and Actb, respectively, in sorted PROCR ${ }^{+}$LT-HSCs from Pten ${ }^{+/+}$and $P$ ten ${ }^{5398 A / 5398 A}$ mice. Each symbol represents data from cells isolated from an individual mouse. In all panels, mean and SEM are shown. ${ }^{*} P<0.05,{ }^{* *} P<0.01,{ }^{* * *} P<0.001$, assessed by $t$ test (A, C, and $\left.\mathbf{D}\right)$ or 1-way ANOVA with Tukey's multiple-comparison test (B). 
A

CD45.2 $2^{+}$Pten $^{+/+}$or Pten ${ }^{S 398 A N S 398 A}$ sorted PROCR ${ }^{+}$LT-HSCs

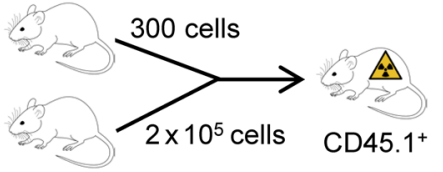

CD45.1+ (wild-type) whole bone marrow
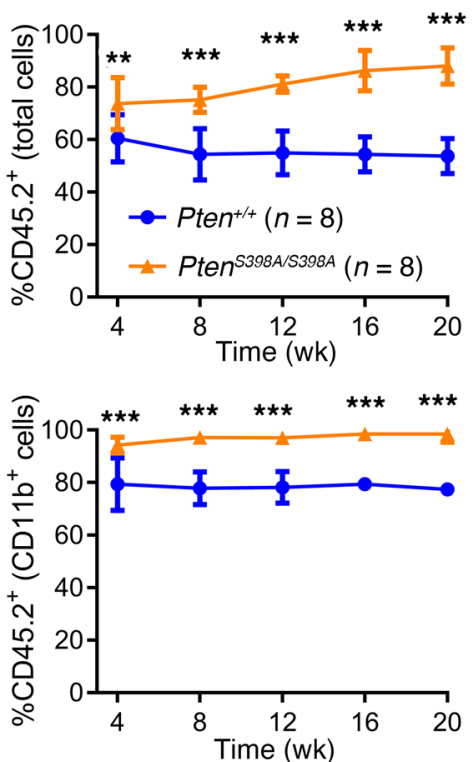
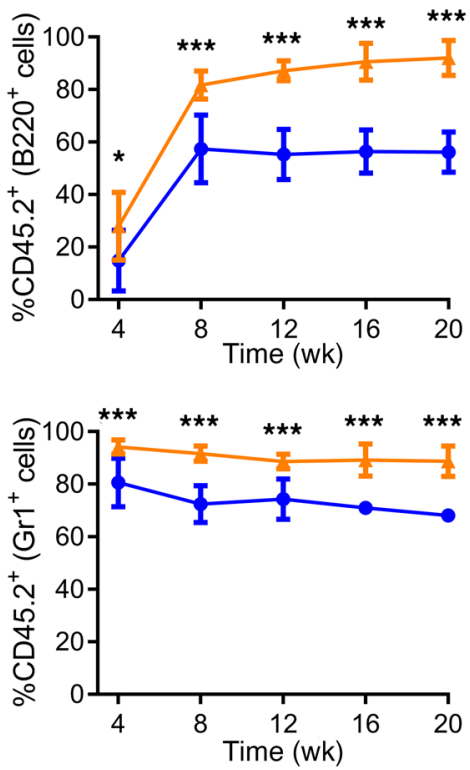

B

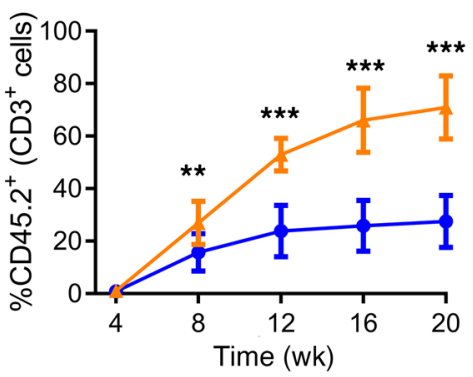

C
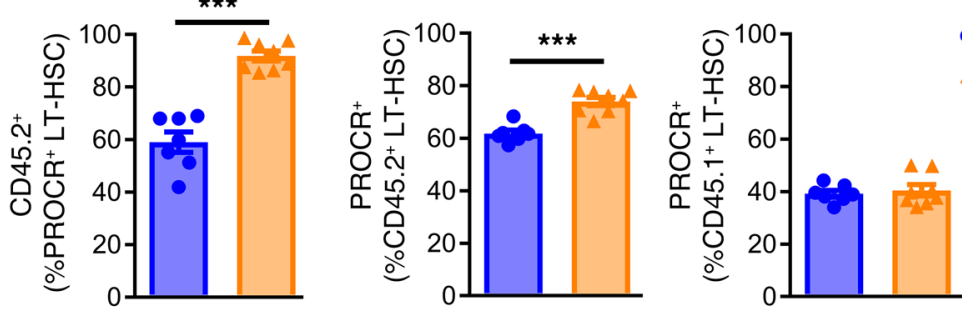

- Pten $^{+/+}$

A Pten ${ }^{\text {S398A/S398a }}$

Figure 7. Improved multilineage hematopoietic reconstitution ability of Pten ${ }^{5398 A / 5398 A}$ PROCR $^{+}$hematopoietic stem cells in vivo. (A) Diagram representing the experimental setup, and plots showing the proportion of circulating total blood cells, B220 ${ }^{+}$cells, CD $3^{+}$cells, CD11 b cells, and Gr1 ${ }^{+}$cells expressing the CD45.2 marker in the peripheral blood of lethally irradiated mice transplanted with 300 Pten $^{+/+}$or Pten ${ }^{5398 A / 5398 A}$ CD 45.2 $2^{+}$PROCR ${ }^{+}$LT-HSCs mixed with 2 $\times 10^{5}$ wild-type CD45.1+ competitors. Transplanted cells were isolated from a pool of 3 donor animals per genotype; " $n$ " indicates the number of transplanted mice. (B) Proportion of LT-HSCs expressing CD45.2 in the bone marrow from mice transplanted as depicted in panel A, analyzed at 20 weeks after transplantation. The gating strategy is depicted in Supplemental Figure 7A. (C) Proportion of CD45.2+ (left) and CD45.1+ (right) LT-HSCs expressing PROCR in the bone marrow from mice transplanted as depicted in panel $\mathbf{A}$, analyzed at 20 weeks after transplantation. In all panels, mean and SEM are shown. ${ }^{*} P$ $<0.05$; ${ }^{*} P<0.01$; ${ }^{* *} P<0.001$; assessed by 2-way ANOVA with Sidak's multiple-comparison test $(\mathbf{A})$ or $t$ test (B and $\mathbf{C}$ ).

identified the genes that were differentially expressed between the 2 cell populations (Supplemental Table 1). GSEA revealed that dormant ${ }^{\text {PTEN-S398A }}$ cells display elements of elevated mitochondrial activity and oxidative phosphorylation, a major source of endogenous ROS (Supplemental Figure 6C). Dormant ${ }^{\mathrm{PTEN}-3398 \mathrm{~A}}$ cells expressed higher levels of mitochondrial function markers (e.g., Atp5h, Cox6c, and Ndufa4) and ROS-sensitive genes (e.g., Prdx1 and $T x n 1$ ) than dormant cells, and on par with the active cells (Figure 5D). This was accompanied by lower expression of Trp53 and of the p53-dependent antioxidant gene Sesn1 (Figure 5D). GSEA further indicated an impaired overall response to oxidative stress in dormant ${ }^{\mathrm{PTEN}-3398 \mathrm{~A}}$ cells, pointing to possible defects in ROS-sensing mechanisms (Supplemental Figure 6C). These observations suggested that $\mathrm{Pten}^{\mathrm{S398A} / 3398 \mathrm{~A}}$ HSCs could aberrantly tolerate elevated mitochondrial activity and ROS-generating oxidative phosphorylation, which are normally associated with a transition from a dormant to an active state (11). We therefore measured ROS levels and mitochondrial content in $\mathrm{Pten}^{+/+}$and $\mathrm{Pten}^{5398 \mathrm{~A} / 3398 \mathrm{~A}}$ dormant HSCs by flow cytometry. Our single-cell data indicated that dormant and dormant ${ }^{\mathrm{PTEN}-\text { S398A }}$ cells could be identified by preferential expression of Procr (Figure 5D), a cell-surface marker of exceptionally multipotent HSCs (34). PROCR ${ }^{+}$LT-HSCs were slightly, but significantly, expanded in Pten ${ }^{3398 A / 3398 A}$ mice compared with their $\mathrm{Pten}^{+/+}$littermates (Figure 6A). $\mathrm{Atm}^{+/-}$mice also had a higher proportion of PROCR ${ }^{+}$LT-HSCs, whereas healthy $\mathrm{Atm}^{-/}$mice were more variable and not significantly different from $\mathrm{Atm}^{+/+}$animals (Figure 6B). Furthermore, Pten ${ }^{5398 A / 3398 A}$ PROCR $^{+}$LT-HSCs had higher intracellular ROS levels and mitochondrial content than their $\mathrm{Pten}^{+/+}$counterparts (Figure 6C). The elevation in mitochondrial content was further confirmed using a qPCR assay to measure the ratio of mitochondrial to nuclear DNA (ref. 35 and Figure 6D).

To verify whether the enhanced reconstitution potential of transplanted Pten ${ }^{S 398 A / 3398 A}$ bone marrow cells was specifically due to improved fitness of quiescent LT-HSCs, we competitively transplanted 300 sorted Pten $^{+/+}$or Pten ${ }^{\text {S398A/3398A }}$ PROCR $^{+}$LT-HSCs (CD45.2+) alongside $2 \times 10^{5}$ wild-type $\mathrm{CD} 45.1^{+}$bone marrow cells into lethally irradiated $\mathrm{CD} 45.1^{+}$recipients (Figure 7A). Compared with $\mathrm{Pten}^{+/}$, Pten $^{5398 A / / 3988 A}$ cells showed improved relative multilineage reconstitution of hematopoiesis (Figure 7A). Twenty weeks after transplantation, we analyzed the bone marrow of recipient 
A

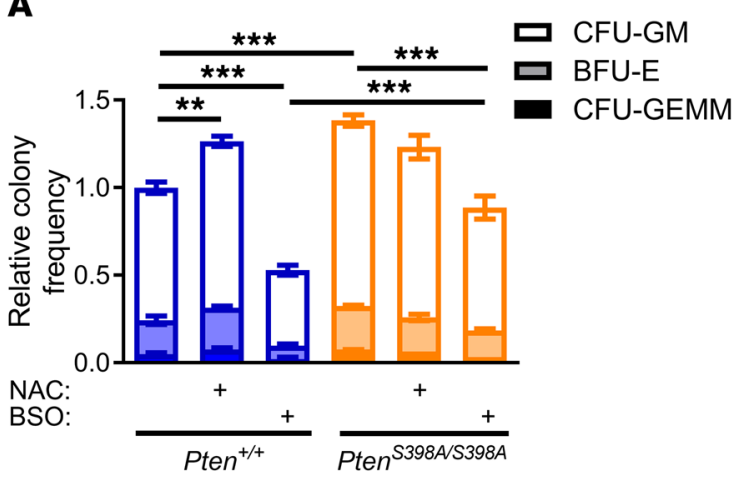

C

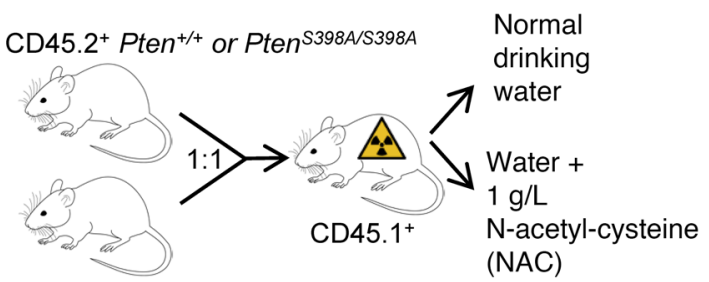

CD45.1+ (wild-type)
B

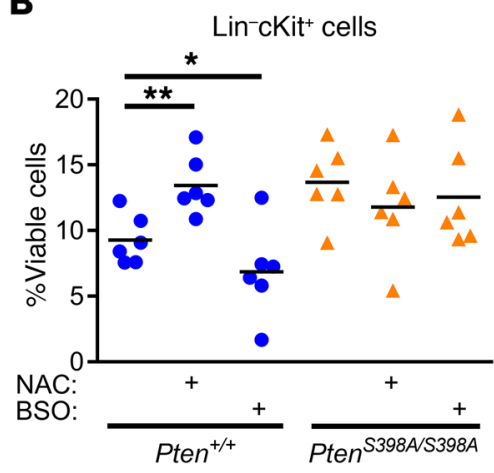

D

$\rightarrow \operatorname{Pten}^{+/+}(n=6) \quad \rightarrow \operatorname{Pten}^{\text {S398A/S398A }}(n=5)$

$\rightarrow$ Pten $^{+/+}+\operatorname{NAC}(n=7)-\operatorname{Pten}^{\text {S398A/S398A }}+\operatorname{NAC}(n=6)$
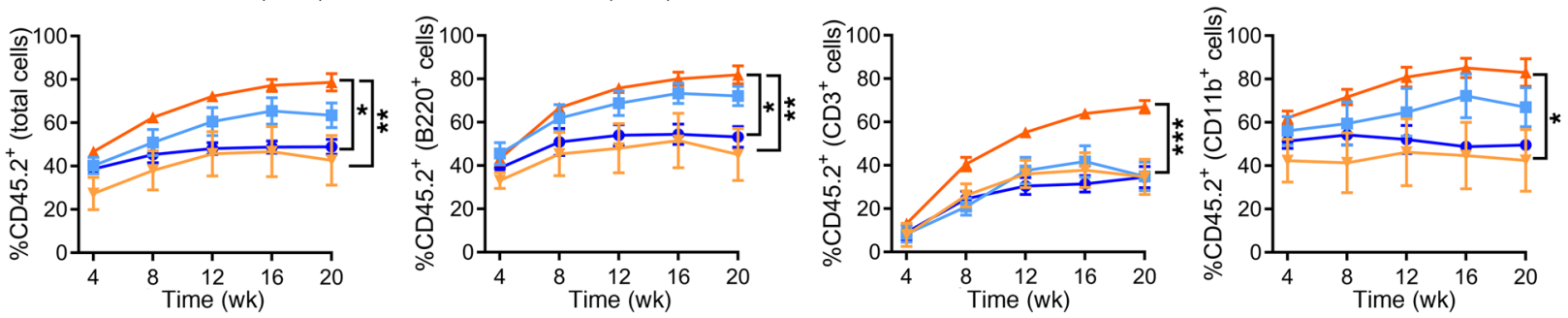

$\mathbf{E}$

$\mathrm{Lin}^{-} \mathrm{CKit}^{+} \mathrm{Sca}{ }^{+}{ }^{+} \mathrm{CD} 48^{-} \mathrm{CD} 150^{+} \mathrm{PROCR}^{+}$
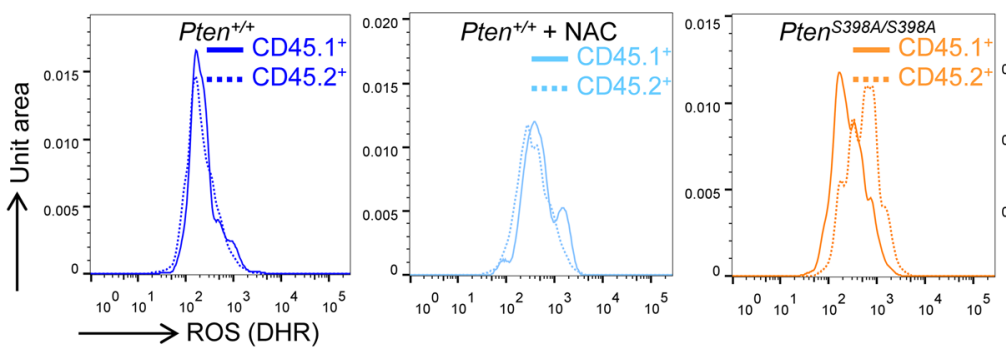

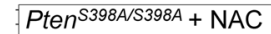

F
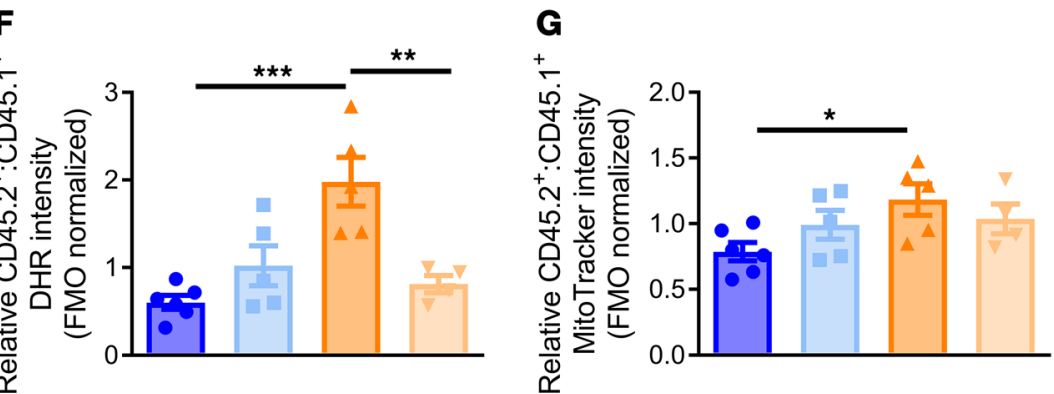

- Pten ${ }^{+/+}$

Pten ${ }^{+/+}+$NAC

- Pten ${ }^{S 398 A / S 398 A}$

$P$ ten ${ }^{\text {S398A/S398A }}+\mathrm{NAC}$ 
Figure 8. Normalization of the competitive fitness of Pten $^{5398 A / 5398 A}$ HSCs by antioxidant treatment. (A) Relative frequency of hematopoietic colonies formed by $\mathrm{Pten}^{+/+}$and $\mathrm{Pten}^{5398 \mathrm{~A} / 5398 \mathrm{~A}}$ bone marrow cells in M3434 methylcellulose medium supplemented or not with $1 \mathrm{mM}$ $N$-acetyl-L-cysteine (NAC) or $1 \mu \mathrm{M}$ buthionine sulfoximine (BSO). $n=6$ mice per genotype. Significance symbols indicate differences in the total number of colonies. CFU-GM, granulocyte/macrophage colony-forming unit; BFU-E, burst-forming unit, erythroid; CFU-GEMM, granulocyte/ erythrocyte/monocyte/megakaryocyte colony-forming unit. (B) Proportion of lineage-negative (Lin-), cKit-positive cells, assessed by flow cytometry, in methylcellulose colonies from the experiments shown in panel $\mathbf{A}$. Each symbol represents a culture from an individual animal. (C) Diagram of the experimental strategy to evaluate the effect of NAC treatment on competitive hematopoietic reconstitution by $\mathrm{Pten}^{+/+}$and $\mathrm{Pten}^{5398 A / 5398 A}$ bone marrow cells. (D) Proportion of circulating total blood cells, B220+ cells, $\mathrm{CD}^{+}$cells, and $\mathrm{CD} 11 \mathrm{~b}^{+}$cells expressing the $\mathrm{CD} 45.2$ marker in the peripheral blood of lethally irradiated mice transplanted as depicted in panel $\mathbf{C}$. Transplanted cells were pooled from 3 donor animals per genotype; " $n$ " indicates the number of transplanted mice. Statistical analyses indicate differences at 20 weeks. (E) Flow cytometry plots depicting dihydrorhodamine (DHR) fluorescence in $\mathrm{Lin}^{-} \mathrm{CKit}{ }^{+} \mathrm{Sca}{ }^{1+C D 48}{ }^{-C D} 150^{+} \mathrm{PROCR}^{+}$cells expressing CD45.1 (wild-type) or CD45.2 (Pten ${ }^{+/+}$or Pten ${ }^{5398 / / 5398 A}$ ) isolated at 20 weeks after transplant. (F) Quantification of the data shown in panel E, expressed as a ratio of the DHR fluorescence in CD45.2+ cells over CD45.1+ cells within each animal. Each symbol represents an individual mouse. (G) Quantification of MitoTracker Green fluorescence in $\mathrm{Lin}^{-} \mathrm{CKit}^{+} \mathrm{Sca}{ }^{+}{ }^{+} \mathrm{CD} 48^{-} \mathrm{CD} 150^{+}{ }^{+} \mathrm{PROCR}{ }^{+}$cells isolated at 20 weeks after transplant, expressed as a ratio of the signal intensity in CD45.2 $2^{+}$cells over CD45.1 $1^{+}$cells within each animal. Each symbol represents an individual mouse. In all panels, mean and SEM are shown. ${ }^{*} P$ $<0.05$; ${ }^{* *} P<0.01$; ${ }^{* *} P<0.001$; assessed by 1-way ANOVA (B, F, and $\mathbf{G}$ ) or 2-way ANOVA (A and $\mathbf{D}$ ) with Tukey's multiple-comparison test.

mice. Compared with $\mathrm{Pten}^{+/+}$recipients, animals transplanted with Pten $^{\mathrm{S398A} / 3398 \mathrm{~A}}$ cells had a higher proportion of CD $45.2^{+} \mathrm{PRO}-$ $\mathrm{CR}^{+}$LT-HSCs (Figure 7B and Supplemental Figure 7A). In addition, the proportion of CD $45.2^{+}$LT-HSCs expressing PROCR was higher in the bone marrow of $P_{t e n}{ }^{5398 A / / 3398 A}$ recipients (Figure 7C). In contrast, the proportion of CD $45.1^{+}$LT-HSCs expressing PROCR was similar between the conditions, as expected given that the transplanted $\mathrm{CD} 45.1^{+}$cells were derived from the same group of wild-type donor mice (Figure 7C). Notably, we observed a similar preferential expansion of PROCR-expressing CD45.2 LT-HSCs in the bone marrow of animals competitively transplanted with wild-type CD $45.1^{+}$and $\mathrm{Atm}^{+-}, \mathrm{Atm}^{-/}$, or IR-exposed Pten $^{\text {S398A } / 3398 A}$ CD $45.2^{+}$cells (Supplemental Figure 7, B and C). Collectively, these results suggest that Pten ${ }^{3398 A / 3398 A}$ quiescent LT-HSCs have improved functional fitness in vivo.

Enhanced competitive fitness of Pten ${ }^{5398 A / 3398 A}$ HSCs is normalized by antioxidant treatment. To functionally test whether the enhanced fitness of Pten ${ }^{5398 A / 3398 A}$ HSCs may be due to their ability to tolerate endogenous oxidative stress, we first measured the colony-forming ability of $\mathrm{Pten}^{+/+}$and $\mathrm{Pten}^{\mathrm{S398A} / 3398 \mathrm{~A}}$ bone marrow cells in methylcellulose cultures in the presence or absence of the antioxidant $\mathrm{N}$-acetyl-L-cysteine (NAC), or the pro-oxidant BSO. Similar to previous results (Figure 3C), Pten ${ }^{5398 A / 3398 A}$ cells had enhanced colony-forming potential compared with their $\mathrm{Pten}^{+/+}$ counterparts (Figure 8A). Addition of NAClead to an increase in the number of colonies in $\mathrm{Pten}^{+/+}$cultures, whereas treatment with BSO had the opposite effect, suggesting that oxidative stress impairs the colony-forming ability of normal cells in these assays (Figure $8 \mathrm{~A})$. In contrast, NAC had no effect on the number of colonies in
Pten $^{\text {S398A } / 3398 A}$ cultures (Figure $8 \mathrm{~A}$ ). BSO treatment decreased the number of colonies generated by Pten $^{\text {S398A/S398A }}$ cells, but it was still substantially higher than in $\mathrm{Pten}^{+/+}$cultures under the same conditions (Figure 8A). We also assessed the differentiation status of colony-forming cells after 7 days of culture. Similar to their effects on the number of colonies, NAC and BSO increased and decreased, respectively, the proportion of $\mathrm{Lin}^{-}, \mathrm{Lin}^{-} \mathrm{cKit}^{+}$, and $\mathrm{Lin}^{-} \mathrm{CKit}^{+} \mathrm{Sca}{ }^{+}$cells in the $\mathrm{Pten}^{+/+}$cultures (Figure 8B and Supplemental Figure 8, A-C). By contrast, Pten ${ }^{\$ 398 A / 3398 A}$ cultures, which contained a higher proportion of Lin $^{-}$cells under basal conditions, were largely insensitive to the effect of NAC or BSO across the $\mathrm{Lin}^{-}, \mathrm{Lin}^{-} \mathrm{CKit}^{+}$, and $\mathrm{Lin}^{-} \mathrm{CKit}^{+} \mathrm{Sca}{ }^{+}$subsets (Figure 8B and Supplemental Figure 8, A-C).

The above results raised the possibility that treatment with antioxidants may equalize the reconstitution potential of $\mathrm{Pten}^{+/+}$ and Pten ${ }^{3398 A / 3398 A}$ cells in bone marrow chimeras. To test this, we competitively transplanted equal numbers of wild-type CD $45.1^{+}$ and $\mathrm{Pten}^{+/+}$or $\mathrm{Pten}^{\mathrm{S} 398 \mathrm{~A} / 3398 \mathrm{C}} \mathrm{CD} 45.2^{+}$bone marrow cells in lethally irradiated $\mathrm{CD} 45.1^{+}$recipients. The transplanted animals were then assigned to either have access to normal drinking water, or to water supplemented with NAC (Figure 8C). Similar to previous experiments, over the course of 20 weeks after transplantation, Pten $^{5398 A / 3398 A} \mathrm{CD} 45.2^{+}$-derived cells displayed better multilineage reconstitution potential than their $\mathrm{Pten}^{+/+} \mathrm{CD} 45.2^{+}$counterparts in mice with normal water (Figure 8D and Supplemental Figure 8D). Importantly, differences between $\mathrm{Pten}^{+/+}$and $\mathrm{Pten}^{\text {S398A//3398A }}$ cells were largely abolished in mice receiving NAC (Figure 8D). The apparent decrease in relative fitness of Pten ${ }^{\text {S398A/3398A }}$ cells in the presence of NAC may reflect a balancing positive effect of antioxidant treatment on the competing CD $45.1^{+}$cells. To relate the effect of NAC to oxidative stress levels within HSCs, we measured intracellular ROS levels in $\mathrm{PROCR}^{+}$LT-HSCs within the bone marrow of transplanted mice. Consistent with the above observations, ROS levels were higher in Pten ${ }^{5398 A / 3398 A} \mathrm{CD} 45.2^{+}$cells compared with their wild-type $\mathrm{CD} 45.1^{+}$competitors, unlike those in mice transplanted with $\mathrm{Pten}^{+/+} \mathrm{CD} 45.2^{+}$cells (Figure 8, E and F). The differences in the relative ROS levels between the competing PROCR ${ }^{+}$ LT-HSC populations were normalized by NAC treatment (Figure 8 F), while similar trends were observed for the mitochondrial content of PROCR ${ }^{+}$LT-HSCs (Figure 8G and Supplemental Figure 8E). Thus, the enhanced function of Pten ${ }^{3398 A / 3398 A}$ cells in bone marrow chimeras may be explained, at least in part, by their enhanced ability to tolerate endogenous oxidative stress.

\section{Discussion}

HSC functionality is tightly dependent on the control of genomic stability, which is extensively governed by ATM. Here, we show that HSCs in which PTEN cannot be phosphorylated by ATM have improved fitness and resistance to genotoxic stress, associated with an altered dormant state. These results suggest that the ATM/PTEN axis forms an essential part of the mechanisms that control HSC dormancy. Our single-cell transcriptomic and functional analyses indicated that a subset of Pten ${ }^{5398 A / 3398 A}$ dormant HSCs can aberrantly tolerate increased ROS levels. A concomitant elevation in mitochondrial activity suggests that ROS accumulation is possibly due to enhanced oxidative phosphorylation. Metabolic activity in HSCs is tightly 
controlled, and increased oxidative phosphorylation is associated with a transition from the dormant to active state $(11,36$, 37). This could form part of the mechanisms that safeguard HSC integrity, by coupling the potential genotoxic effect of ROS to differentiation priming. Indeed, studies in multiple mouse models have identified ROS elevation as a main cause of HSC dysfunction following disruption of regulators of the DNA damage response $(15,19,38-40)$. We speculate that an abnormal response to ROS in Pten ${ }^{S 398 A / S 398 A}$ HSCs may enable some Pten $^{\text {S398A/S398A }}$ dormant HSCs to acquire the biosynthetic capabilities of active cells, while retaining the quiescence and cellular characteristics necessary to maintain their multipotency (Supplemental Figure 9). Such a mechanism, which remains to be formally tested, could conceivably endow Pten ${ }^{5398 A / S 398 A}$ HSCs with superior fitness, as we observe in competitive transplantation assays. In support of this possibility, a recent study has demonstrated that elevated mitochondrial activity enhances the functionality of aged HSCs (41). It would be interesting to investigate whether this phenomenon is associated with an altered response to oxidative stress, coupled with the reported resistance of aged HSCs to genotoxins (42). Furthermore, PTEN itself is sensitive to the cellular redox state (43-45), and it is possible that the Pten ${ }^{5398 A}$ mutation could disrupt this regulation. Additional alterations in Pten ${ }^{5398 A / S 398 A}$ HSCs, such as defective apoptotic priming in response to DNA damage, may also contribute to improved HSC functionality by permitting the survival of HSCs that would normally be eliminated.

PTEN can localize to multiple subcellular compartments, where it exerts diverse functions (23). Previous work has shown that PTEN is excluded from the nucleus in response to genotoxic stress and phosphorylation on S398 by ATM (21). In the present study, we observed a similar redistribution of PTEN in HSCs following IR, which was impaired by the Pten $^{\text {S398A }}$ mutation. Such exclusion of PTEN from the nucleus could enhance its activity in other compartments, including at the plasma membrane where it functions as a lipid phosphatase to negatively regulate AKT signaling $(46,47)$. This may affect multiple AKT-dependent signaling pathways that control HSC function and response to genotoxic stress, including mTOR complexes and FOXO transcription factors (24, 48-51). The cellular response to signals from the microenvironment that are transduced via AKT signaling, such as SCF, may be modulated by the subcellular distribution of PTEN, potentially contributing to the control of HSC quiescence and fitness. Future studies could elucidate the dynamics of PTEN nuclear-cytoplasmic shuttling in homeostasis and in response to DNA damage in HSCs, preferably within their native bone marrow environment. Although we noted several similarities between the $\mathrm{Atm}^{+/-}$and $\mathrm{Pten}^{\mathrm{S398A} / \mathrm{S3} 98 \mathrm{~A}}$ genotypes, given the multiple targets of ATM, it is possible that some effects of Atm heterozygosity are mediated independently of PTEN regulation. Among several other processes, ATM has been implicated in the regulation of autophagy, metabolism, and the response to replication stress (52-55), all of which are important to control HSC fitness $(37,56,57)$. Whether some of these functions involve ATM-mediated PTEN phosphorylation will be an interesting topic for further investigations.
The phenotype of Pten ${ }^{\text {S398A/S398A }}$ HSCs bears some resemblance to aging HSCs, which preserve enhanced functionality compared with young HSCs in response to DNA-damaging insults (42). Interestingly, this property of aging HSCs is at least partially dependent on reduced ATM function (42), which could be recapitulated by loss of ATM-dependent PTEN regulation in our Pten ${ }^{S 398 A / S 398 A}$ model, as well as in $\mathrm{Atm}^{+/-}$mice. A drawback of higher tolerance to genotoxic stress might be an increased susceptibility to acquire oncogenic lesions (58). We did not observe higher incidence of spontaneous hematologic malignancies throughout the lifespan of $P t e n^{S 398 A / S 398 A}$ mice, or in bone marrow chimeras transplanted with Pten $^{\$ 398 A / S 398 A}$ cells, suggesting that additional oncogenic hits may be required to uncover a possible tumor-promoting effect of the Pten ${ }^{\$ 398 A}$ mutation in the hematopoietic system. Furthermore, previous work has shown that, by promoting differentiation, disruption of the DNA damage response in hematopoietic cells (including by ATM inhibition or ablation) can have a paradoxical tumor-protective effect (39, 59). These observations highlight the complex role of the DNA damage response in controlling homeostasis and tumorigenesis in the hematopoietic system.

Overall, our studies identify ATM phosphorylation of PTEN as essential to safeguard HSC dormancy and response to genotoxic stress. These results could inform strategies for hematopoietic regeneration, and for anticancer treatments in hematologic malignancies.

\section{Methods}

Detailed descriptions of animals and experimental procedures are provided in Supplemental Methods.

Study approval. All animal experiments were performed in accordance with institutional and federal guidelines, and approved by the institutional Animal Care Committee (protocols 985 and 5975).

Publicly deposited data and accession numbers. The single-cell RNA-seq data have been deposited in NCBI's Gene Expression Omnibus database (GEO GSE164388).

\section{Author contributions}

JF and CB conceived the study, designed and performed experiments, analyzed the results, and wrote the manuscript with input from all the authors. PR performed single-cell bioinformatics analyses. WYL designed and performed single-cell transcriptomic experiments. RT, IZ, and GH performed experiments and analyzed the results. BES, JH, CT, $\mathrm{KH}$, and $\mathrm{AW}$ generated mice and performed experiments. VS and TWM conceived and supervised the study. All authors revised and approved the manuscript.

\section{Acknowledgments}

This study was supported by a Canadian Institutes of Health Research (CIHR) Foundation Grant to TWM. JF was supported by a fellowship from the CIHR. The authors thank the flow cytometry facility at Princess Margaret Hospital and the Princess Margaret Genomics Centre for support.

Address correspondence to: Tak W. Mak, 610 University Avenue, Room 9-406, Toronto, Ontario M5G 2M9, Canada. Phone: 416.946.2234; Email:tmak@uhnresearch.ca. 
1. Ades L, et al. Myelodysplastic syndromes. Lancet. 2014;383(9936):2239-2252.

2. Ceccaldi R, et al. The Fanconi anaemia pathway: new players and new functions. Nat Rev Mol Cell Biol. 2016;17(6):337-349.

3. Joenje H, Patel KJ. The emerging genetic and molecular basis of Fanconi anaemia. Nat Rev Genet. 2001;2(6):446-457.

4. Orkin SH, Zon LI. Hematopoiesis: an evolving paradigm for stem cell biology. Cell. 2008;132(4):631-644.

5. Shimamura A, Alter BP. Pathophysiology and management of inherited bone marrow failure syndromes. Blood Rev. 2010;24(3):101-122.

6. Sperling AS, et al. The genetics of myelodysplastic syndrome: from clonal haematopoiesis to secondary leukaemia. Nat Rev Cancer. 2017;17(1):5-19.

7. Seita J, Weissman IL. Hematopoietic stem cell: self-renewal versus differentiation. Wiley Interdiscip Rev Syst Biol Med. 2010;2(6):640-653.

8. Wilson A, et al. Hematopoietic stem cells reversibly switch from dormancy to selfrenewal during homeostasis and repair. Cell. 2008;135(6):1118-1129.

9. Becker AJ, et al. Cytological demonstration of the clonal nature of spleen colonies derived from transplanted mouse marrow cells. Nature. 1963;197:452-454.

10. McCulloch EA, Till JE. The radiation sensitivity of normal mouse bone marrow cells, determined by quantitative marrow transplantation into irradiated mice. Radiat Res. 1960;13:115-125.

11. Cabezas-Wallscheid N, et al. Vitamin A-retinoic acid signaling regulates hematopoietic stem cell dormancy. Cell. 2017;169(5):807-823.

12. Beerman I. Accumulation of DNA damage in the aged hematopoietic stem cell compartment. Semin Hematol. 2017;54(1):12-18.

13. Rossi DJ, et al. Deficiencies in DNA damage repair limit the function of haematopoietic stem cells with age. Nature. 2007;447(7145):725-729.

14. Shiloh Y, Ziv Y. The ATM protein kinase: regulating the cellular response to genotoxic stress, and more. Nat Rev Mol Cell Biol. 2013;14(4):197-210.

15. Ito K, et al. Regulation of oxidative stress by ATM is required for self-renewal of haematopoietic stem cells. Nature. 2004;431(7011):997-1002.

16. Barlow C, et al. Atm-deficient mice: a paradigm of ataxia telangiectasia. Cell. 1996;86(1):159-171.

17. Akala OO, et al. Long-term haematopoietic reconstitution by $\operatorname{Trp} 53^{-/-} \mathrm{p} 16 \operatorname{Ink} 4 \mathrm{a}^{-/}$ p19Arf ${ }^{/}$multipotent progenitors. Nature. 2008;453(7192):228-232.

18. Liu Y, et al. p53 regulates hematopoietic stem cell quiescence. Cell Stem Cell. 2009;4(1):37-48.

19. Maryanovich M, et al. The ATM-BID pathway regulates quiescence and survival of haematopoietic stem cells. Nat Cell Biol. 2012;14(5):535-541.

20. Takai H, et al. Chk2-deficient mice exhibit radioresistance and defective p53-mediated transcription. EMBO J. 2002;21(19):5195-5205.

21. Bassi C, et al. Nuclear PTEN controls DNA repair and sensitivity to genotoxic stress. Science. 2013;341(6144):395-399.
22. Lee YR, et al. The functions and regulation of the PTEN tumour suppressor: new modes and prospects. Nat Rev Mol Cell Biol. 2018;19(9):547-562.

23. Bassi C, Stambolic V. PTEN, here, there, everywhere. Cell Death Differ. 2013;20(12):1595-1596.

24. Yilmaz OH, et al. Pten dependence distinguishes haematopoietic stem cells from leukaemiainitiating cells. Nature. 2006;441(7092):475-482.

25. Zhang J, et al. PTEN maintains haematopoietic stem cells and acts in lineage choice and leukaemia prevention. Nature. 2006;441(7092):518-522.

26. Keller G, Snodgrass R. Life span of multipotential hematopoietic stem cells in vivo. J Exp Med. 1990;171(5):1407-1418.

27. Szilvassy SJ, et al. Quantitative assay for totipotent reconstituting hematopoietic stem cells by a competitive repopulation strategy. Proc Natl Acad Sci U S A. 1990;87(22):8736-8740.

28. Hirao A, et al. DNA damage-induced activation of 53 by the checkpoint kinase Chk2. Science. 2000;287(5459):1824-1827.

29. Louria-Hayon I, et al. Lnk adaptor suppresses radiation resistance and radiation-induced B-cell malignancies by inhibiting IL-11 signaling. Proc Natl Acad Sci U S A. 2013;110(51):20599-20604.

30. Satija R, et al. Spatial reconstruction of singlecell gene expression data. Nat Biotechnol. 2015;33(5):495-502.

31. Scialdone A, et al. Computational assignment of cell-cycle stage from single-cell transcriptome data. Methods. 2015;85:54-61.

32. Giladi A, et al. Single-cell characterization of haematopoietic progenitors and their trajectories in homeostasis and perturbed haematopoiesis. Nat Cell Biol. 2018;20(7):836-846.

33. Wilson NK, et al. Combined single-cell functional and gene expression analysis resolves heterogeneity within stem cell populations. Cell Stem Cell. 2015;16(6):712-724.

34. Balazs AB, et al. Endothelial protein $C$ receptor (CD201) explicitly identifies hematopoietic stem cells in murine bone marrow. Blood. 2006;107(6):2317-2321.

35. de Almeida MJ, et al. Dye-independent methods reveal elevated mitochondrial mass in hematopoietic stem cells. Cell Stem Cell. 2017;21(6):725-729.

36. Simsek T, et al. The distinct metabolic profile of hematopoietic stem cells reflects their location in a hypoxic niche. Cell Stem Cell. 2010;7(3):380-390.

37. Wang YH, et al. Cell-state-specific metabolic dependency in hematopoiesis and leukemogenesis. Cell. 2014;158(6):1309-1323.

38. Liu J, et al. Bmi1 regulates mitochondrial function and the DNA damage response pathway. Nature. 2009;459(7245):387-392.

39. Santos MA, et al. DNA-damage-induced differentiation of leukaemic cells as an anti-cancer barrier. Nature. 2014;514(7520):107-111.

40. Tasdogan A, et al. DNA damage-induced HSPC malfunction depends on ROS accumulation downstream of IFN-1 signaling and bid mobilization. Cell Stem Cell. 2016;19(6):752-767.

41. Mansell E, et al. Mitochondrial potentiation ameliorates age-related heterogeneity in hematopoietic stem cell function. Cell Stem Cell. 2021;28(2):241-256.

42. Gutierrez-Martinez P, et al. Diminished apoptotic priming and ATM signalling confer a survival advantage onto aged haematopoietic stem cells in response to DNA damage. Nat Cell Biol. 2018;20(4):413-421.

43. Kwon J, et al. Reversible oxidation and inactivation of the tumor suppressor PTEN in cells stimulated with peptide growth factors. Proc Natl Acad Sci US A. 2004;101(47):16419-16424.

44. Lee SR, et al. Reversible inactivation of the tumor suppressor PTEN by H2O2. J Biol Chem. 2002;277(23):20336-20342.

45. Leslie NR, et al. Redox regulation of PI 3-kinase signalling via inactivation of PTEN. EMBO J. 2003;22(20):5501-5510.

46. Stambolic V, et al. Negative regulation of PKB/ Akt-dependent cell survival by the tumor suppressor PTEN. Cell. 1998;95(1):29-39.

47. Lee JO, et al. Crystal structure of the PTEN tumor suppressor: implications for its phosphoinositide phosphatase activity and membrane association. Cell.1999;99(3):323-334.

48. Chen C, et al. mTOR regulation and therapeutic rejuvenation of aging hematopoietic stem cells. Sci Signal. 2009;2(98):ra75.

49. Miyamoto K, et al. Foxo3a is essential for maintenance of the hematopoietic stem cell pool. Cell Stem Cell. 2007;1(1):101-112.

50. Rimmele P, et al. Mitochondrial metabolism in hematopoietic stem cells requires functional FOXO3. EMBO Rep. 2015;16(9):1164-1176.

51. Tothova Z, et al. FoxOs are critical mediators of hematopoietic stem cell resistance to physiologic oxidative stress. Cell. 2007;128(2):325-339.

52. Aird KM, et al. ATM couples replication stress and metabolic reprogramming during cellular senescence. Cell Rep. 2015;11(6):893-901.

53. Dahl ES, Aird KM. Ataxia-telangiectasia mutated modulation of carbon metabolism in cancer. Front Oncol. 2017;7:291.

54. Olcina MM, et al. Replication stress and chromatin context link ATM activation to a role in DNA replication. Mol Cell. 2013;52(5):758-766.

55. Stagni V, et al. Ataxia-telangiectasia mutated kinase in the control of oxidative stress, mitochondria, and autophagy in cancer: a maestro with a large orchestra. Front Oncol. 2018;8:73.

56 . Flach J, et al. Replication stress is a potent driver of functional decline in ageing haematopoietic stem cells. Nature. 2014;512(7513):198-202.

57. Ho TT, et al. Autophagy maintains the metabolism and function of young and old stem cells. Nature. 2017;543(7644):205-210.

58. Beerman I, et al. Quiescent hematopoietic stem cells accumulate DNA damage during aging that is repaired upon entry into cell cycle. Cell Stem Cell. 2014;15(1):37-50.

59. Morgado-Palacin I, et al. Targeting the kinase activities of ATR and ATM exhibits antitumoral activity in mouse models of MLL-rearranged AML. Sci Signal. 2016;9(445):ra91. 